\title{
Physiology-based toxicokinetic modeling in the frame of the European Human Biomonitoring Initiative
}

Dimosthenis A. Sarigiannis ${ }^{1,2^{*}}$, Spyros Karakitsios ${ }^{1,2}$, Elena Dominguez-Romero ${ }^{3}$, Krystalia Papadaki ${ }^{1}$, Celine Brochot ${ }^{4}$, Vikas Kumar ${ }^{5}$, Marta Schumacher ${ }^{5}$, Moustapha $\mathrm{Sy}^{6}$, Hans Mielke ${ }^{6}$, Mathias Greiner ${ }^{6}$, Marcel Mengelers ${ }^{7}$, Martin Scheringer ${ }^{3}$

${ }^{1}$ Aristotle University of Thessaloniki, Department of Chemical Engineering, Environmental Engineering Laboratory, University Campus, Thessaloniki 54124, Greece

${ }^{2}$ HERACLES Research Center on the Exposome and Health, Center for Interdisciplinary Research and Innovation, Balkan Center, Bldg. B, $10^{\text {th }} \mathrm{km}$ Thessaloniki-Thermi Road, 57001, Greece

${ }^{3}$ RECETOX, Masaryk University, Brno, Czech Republic

${ }^{4}$ INERIS, France

${ }^{5}$ IISPV, Tarragona, Spain

${ }^{6} \mathrm{BfR}$, Berlin, Germany

${ }^{7}$ RIVM, Ultrecht, The Netherlands

${ }^{*}$ Corresponding author: Environmental Engineering Laboratory, Department of Chemical Engineering, Aristotle University of Thessaloniki, University Campus, Bldg. D, Rm 201, 54124 Thessaloniki, Greece; Tel. +30-2310-994562; denis@eng.auth.gr 


\section{Abstract}

Given the opportunities provided by internal dosimetry modelling in the interpretation of human biomonitoring (HBM) data, the assessment of the links between exposure to chemicals and observed HBM data can be effectively supported by PBTK modelling. This paper gives a comprehensive review of available human PBTK models for compounds selected as a priority by the European Human Biomonitoring Initiative (HBM4EU). We highlight their advantages and deficiencies and suggest steps for advanced internal dose modelling. The review of the available PBTK models highlighted the conceptual differences between older models compared to the ones developed recently, reflecting commensurate differences in research questions. Due to the lack of coordinated strategies for deriving useful biomonitoring data for toxicokinetic properties, significant problems in model parameterisation still remain; these are further increased by the lack of human toxicokinetic data due to ethics issues.

Finally, questions arise as well as to the extent they are really representative of interindividual variability. QSARs for toxicokinetic properties is a complementary approach for PBTK model parameterisation, especially for data poor chemicals. This approach could be expanded to model chemico-biological interactions such as intestinal absorption and renal clearance; this could serve the development of more complex generic PBTK models that could be applied to newly derived chemicals.

Another gap identified is the framework for mixture interaction terms among compounds that could eventually interact in metabolism. From the review it was concluded that efforts should be shifted toward the development of generic multi-compartmental and multi-route models, supported by targeted biomonitoring coupled with parameterisation by both QSAR approach and experimental (in-vivo and in-vitro) data for newly developed and data poor compounds.

\section{Abbreviations}

2,6-DMA; 2,6-Dimethoxyamphetamine, 3-OH-BaP; 3-hydroxybenzo(a)pyrene, 5-cx MEPP ; Mono(2-ethyl5-carboxypentyl) Phthalate, 5-OH MEHP; Mono-(2-ethyl-5-hydroxyhexyl) Phthalate, 5-oxo-MEHP; Mono(2-ethyl-5-oxohexyl) Phthalate, ACAT ; Advanced Compartmental Absorption and Transit model, AChE; Acetylcholinesterase, ADME; Absorption, Distribution, Metabolism, Excretion, AFE; Average Fold Error, AUC; Area Under the Curve , BaP; Benzo(a)pyrene, BD; 1,3-butadiene, BDE-47; 2,2',4,4'tetrabromodiphenyl ether, BDEs; Brominated Diphenyl Ethers, BFRs; Brominated Flame Retardants, BMI; Body Mass Index, BPA; Bisphenol A, BPAG; Bisphenol A Glucuronide, BPAS; Bisphenol A Sulfate, BPF; Bisphenol F, BPS; Bisphenol S, BTEX; Benzene, Toluene, Ethylbenzene, Xylene, BuChE; Butyrylcholinesterase, BW; Body Weight, CAE; Chloracetate Esterase, ChE; Cholinesterase, CO; Cardiac Output, CPF; Chlorpyrifos, CV; Coefficient of Variation, DEHP; Di(2-ethylhexyl) Phthalate, DINCH; 1,2cyclohexane dicarboxylic acid diisononyl ester, DMA; Dimethylarsinic acid, DMT-1; Divalent Metal Transporter 1, DnBP; Di-n-Butyl Phthalate, EHR; Enterohepatic Recirculation, EPA; Environmental Protection Agency, ER; Estrogen Receptor, ERDEM; Exposure Related Dose Estimating Model, FDA; Food and Drug Administration, GEC; Gastric Emptying Constant, GFR; Glomerular Filtration Rate, Gl; Gastrointestinal, HBM ; Human Biomonitoring, IV; Intravenous, IVIVE; In Vitro to In Vivo Extrapolation, MAPE; Median Absolute Performance Error, MBP; Monobutyl Phthalate, MBP-G ; Monobutyl Phthalate 
Glucuronide, MC; Markov Chain, MCMC; Markov Chain Monte Carlo, MDSs; Model Data Sets, MEHP; Mono(2-ethylhexyl) Phthalate, MLR; Multiple Linear Regression, MMA; Methylarsonic Acid, MMAD; Mass Median Aerodynamic Diameter, MOCA; 4,4'-Methylenebis(2-chloroaniline), MRD; Mean Relative Deviation, MSP; Microsomal Protein, MTBE; Methyltert-butylether, NHANES; National Health and Nutrition Examination Surveys, NHEXAS; National Human Exposure Assessment Survey, NMP; N-methylpyrrolidone, NSC; Normalised Sensitivity Coefficient, OP; Organophosphate, o-TOL; o-toluidine, PAHs; Polycyclic Aromatic Hydrocarbons, PBBK ; Physiologically Based Biokinetic models, PBPK; Physiologically Based Pharmacokinetic models, PBTK; Physiologically Based Toxicokinetic models, PC; Partition Coefficient, PCBs; Polychlorinated Biphenyls, PCDDs; Polychlorinated Dibenzo-p-Dioxins, PE;

Performance Error, PFAA; Perfluoroalkyl acid, PFASs; Per- and polyfluoroalkyl substances, PFCs; Perfluorinated Compounds, PFOA; Perfluorooctanoic acid, PFOS; Perfluorooctanesulfonic acid, P-PBPK ; Pregnancy-Physiologically Based Pharmacokinetic model, QSARs; Quantitative Structure-Activity Relationships, QSPRs; Quantitative Structure-Property Relationships, RMSEP; Root Mean Square Error of Prediction, SD; Standard Deviation, SHEDS-HT; Stochastic Human Exposure and Dose Simulation-High Throughput, SR; Sensitivity Ratio, SRCs; Standardised Regression Coefficients, SVHC; Substances of Very High Concern, TCDD; 2,3,7,8-tetrachlorodibenzo-p-dioxin, TCP; 3,5,6-trichloropyridinol, TCPY; 3,5,6trichloro-2-pyridinol, TLV; Threshold Limit Value, UGT2B7; UDP-Glucuronosyltransferase-2B7, UWW; Uterine Wet Weight, VOCs ; Volatile Organic Compounds

Keywords: PBTK modelling, internal dose, human biomonitoring, HBM4EU 


\section{Introduction}

PBTK models are continuously gaining ground in regulatory toxicology, describing in quantitative terms the absorption, metabolism, distribution and elimination processes in the human body, with a focus on the effective dose at the expected target site (Bois et al., 2010). Considering the growing interest that PBTK models find in the risk assessment process, guidelines for proper species, doses, and exposure scenarios extrapolations have been proposed by Clewell and Clewell (2008), while the steps towards good modelling practice have been explicitly described by Loizou et al. (2008). The need for the widespread use of PBTK models development is further amplified by the increasing scientific and regulatory interest about aggregate and cumulative exposure. PBTK models translate external exposures from multiple routes (Yang et al., 2010) into internal exposure metrics, addressing the importance of different exposure routes in the overall bioavailability (Sarigiannis and Karakitsios, 2011; Valcke and Krishnan, 2011) or the dependence on critical developmental windows of susceptibility, such as pregnancy (Beaudouin et al., 2010), lactation (Verner et al., 2008) and infancy (Edginton and Ritter, 2009b). With regard to cumulative exposure, PBTK models offer a framework for including the effect of the interactions among the mixture compounds on metabolism. However, due to inherent difficulties, the existing applications are currently limited mainly to VOCs (Haddad et al., 2000; Sarigiannis and Gotti, 2008) and metals (Sasso et al., 2010). Recently, efforts have shifted towards the integration of whole-body physiology, disease biology, and molecular reaction networks (Eissing et al., 2011), as well as integration of cellular metabolism into multi-scale whole-body models (Krauss et al., 2012).

The use of internal dose modeling aims at integrating exposure data and modeling output with Human Biomonitoring data. Its goals are to (a) provide the time history of the exposure profile, focusing on susceptible developmental stages; (b) assimilate biomonitoring data to estimate the individual exposome; and (c) derive reliable biologically effective dose values for the compounds of interest so that they can be associated to observed health outcomes. The key component of the above is the development of a lifetime (including gestation and breastfeeding) generic PBTK model (Sarigiannis and Karakitsios, 2012; Sarigiannis et al., 2016a) incorporating mixture interaction (Sarigiannis and Gotti, 2008) and a framework for biomonitoring data assimilation (Georgopoulos et al., 2008a). Aiming to expand the applicability of the generic PBTK model, parameterisation of the model for known and new chemicals with limited information is done through the development of QSAR models.

A generic PBTK model could also be used to reconstruct exposure from Human Biomonitoring data (Andra et al., 2015). Towards this aim, a tiered approach would be followed as a function of data availability (periodicity and size of sampling, specimen type) and requirements of the exposure reconstruction analysis (temporal analysis of exposure, contribution from different routes), ranging from Exposure Conversion Factors (Tan et al., 2006), up to Markov Chain Monte Carlo analysis. Inputs involve spatial and temporal information on media concentrations of xenobiotics and corresponding information on human activities, food intake patterns or consumer product use. Outputs are the observed biomarkers. The error metric can be defined in terms of population variation (the latter has to be lower than the intra-individual variation, which may be associated with measurement or other random error source). PBTK could be combined with multimedia models and survey questionnaires to identify exposure sources. PBTK modeling may also be used to estimate the internal doses of xenobiotics that exceed levels 
associated with biological alterations (Judson et al., 2011) and, eventually, health risk. The latter can involve the use of specific omics results (e.g., metabolomics analysis) and associations of biologically effective doses to early biological responses (toxicodynamics). In addition, biologically effective doses would be used to quantify the effect of compound-induced extracellular perturbations on metabolism, so as to directly couple the PBTK model with metabolic regulatory networks. Direct coupling defines a feedback loop that connects clearance and metabolite production rates to metabolism regulation (Eissing et al., 2011) via dynamic flux balance analysis (Krauss et al., 2012).

Considering the opportunities offered by the use of PBTK models in exposure/risk characterisation, several research groups are developing generic PBTK models, either as stand-alone models such as PK-Sim (Willmann et al., 2003a), Indus-Chem (Jongeneelen and Berge, 2011) or PoPGen (McNally, 2014), or incorporated within integrated computational platforms for exposure assessment such as INTERA (Sarigiannis et al., 2011) and MENTOR (Georgopoulos et al., 2008b). The development of generic PBTK models is substantiated by recent advances in quantitative structure-activity relationships (QSARs) and quantitative structure-property relationships (QSPRs) (Peyret and Krishnan, 2011; Price and Krishnan, 2011), providing the basis for development of relevant PBTK models for data-poor or new chemicals (Papadaki et al., 2017; Sarigiannis et al., 2017).

Recognising key gaps in the knowledge required to support actions on chemicals within the 7th Environmental Action Programme, as well as the need to address these gaps Human Biomonitoring (HBM) was identified as a tool that can serve the chemicals agenda. As a response to these needs, the European Human Biomonitoring Initiative ${ }^{1}$ (HBM4EU) aims at generating knowledge to inform the safe management of chemicals and so protect human health (Ganzleben et al., 2017). HBM4EU represents a joint effort of 28 countries, the European Environment Agency and the European Commission, co-funded by Horizon 2020. The main aim of the initiative is to coordinate and advance human biomonitoring in Europe. HBM4EU provides evidence of the actual exposure of citizens to chemicals and the possible health effects to support policy making. The project involves collaboration between several Commission services, EU agencies, national representatives, stakeholders and scientists, demonstrating how research funding can build bridges between the research and policy worlds. HBM4EU launched on 1 January 2017 and will run until 31 December 2021. HBM4EU will contribute to the overarching goal of ensuring that EU chemical policies minimise the adverse effects of chemicals on human health. An HBM4EU aim, is the acquisition of HBM data on the exposure of the EU population to prioritised chemicals, and to derive European Health based guidance values and statistically derived reference values for bisphenols $(A, S, F)$, phthalates and DiNCH, new generation flame retardants, cadmium, chromium, PAHs, Anillin derivates, and short chain perfluorinated compounds in a first round.

Given the opportunities provided by internal dosimetry modelling in the interpretation of HBM data, the assessment of the links between exposure to chemicals and observed HBM data can be effectively supported by PBTK modelling. To summarise, the aim of this paper is to provide a comprehensive review of human PBTK models available for the HBM4EU prioritised

\footnotetext{
${ }^{1}$ This project has received funding from the European Unions' Horizon 2020 research and innovation Programme under grant agreement No 733032 HBM4EU (www.HBM4EU.eu).
} 
compounds, to highlight their advantages and deficits and to provide steps for increasing the predicting accuracy.

\section{Methodology}

\subsection{Selection of priority compounds}

The compounds that will be dealt with priority in the HBM4EU project, were selected in consortium with primarily policy makers and regulators in chemical/food safety and occupational health in European Union Member States, European institutions responsible for policy making and agencies responsible for implementing EU regulations. The criteria used comprised public concern, scientific evidence and urgency to regulate in order to safeguard occupational and consumer health while fostering industrial competitiveness and innovation.

Keywords

Physiologically based pharmacokinetic (or toxicokinetic or biokinetic) model + PBPK (or PBTK or PBBK) + anilines + human

Physiologically based pharmacokinetic (or toxicokinetic or biokinetic) model + PBPK (or PBTK or PBBK) + bisphenols + human

Physiologically based pharmacokinetic (or toxicokinetic or biokinetic) model + PBPK (or PBTK or PBBK) + bisphenol A + BPA + human

Physiologically based pharmacokinetic (or toxicokinetic or biokinetic) model + PBPK (or PBTK or PBBK) + bisphenol S + BPS + human

Physiologically based pharmacokinetic (or toxicokinetic or biokinetic) model + PBPK (or PBTK or PBBK) + bisphenol F + BPF + human

Physiologically based pharmacokinetic (or toxicokinetic or biokinetic) model + PBPK (or PBTK or PBBK) + phthalates + human

Physiologically based pharmacokinetic (or toxicokinetic or biokinetic) model + PBPK (or PBTK or PBBK) + pahs + human

Physiologically based pharmacokinetic (or toxicokinetic or biokinetic) model + PBPK (or PBTK or PBBK) + polycyclic aromatic hydrocarbons + human

Physiologically based pharmacokinetic (or toxicokinetic or biokinetic) model + PBPK (or PBTK or $\mathrm{PBBK})+$ pesticides + human

Physiologically based pharmacokinetic (or toxicokinetic or biokinetic) model + PBPK (or PBTK or PBBK) + perfluorinated compounds + human

Physiologically based pharmacokinetic (or toxicokinetic or biokinetic) model + PBPK (or PBTK or PBBK) + PFOA + PFOS + human 
Physiologically based pharmacokinetic (or toxicokinetic or biokinetic) model + PBPK (or PBTK or PBBK) + metals + human

Physiologically based pharmacokinetic (or toxicokinetic or biokinetic) model + PBPK (or PBTK or PBBK) + chromium + human

Physiologically based pharmacokinetic (or toxicokinetic or biokinetic) model + PBPK (or PBTK or PBBK) + cadmium + human

Generic + Physiologically based pharmacokinetic (or toxicokinetic or biokinetic) model + PBPK (or PBTK or PBBK) + human

The wide spectrum of chemicals that were proposed as candidates to consider in the frame of the European Human Biomonitoring initiative were classified in three clusters on the basis of the criteria outlined above. Group A includes compounds for which there is enough information, policy relevance, regulatory expediency and/or public concern to warrant successful collection and interpretation of biomonitoring data in Europeans. Group B includes compounds for which data gaps were identified already during preliminary prioritisation but public concern is such that warrants focus right after Group A. Group C includes substances for which the available information is limited and there is no significant urgency to deal with. During the initial 2-3 years of HBM4EU the project puts its main focus on the chemicals in Group A. These include phthalates and $\mathrm{DiNCH}$, anilines and MOCHA, bisphenols, polyaromatic hydrocarbons and airborne chemicals, flame retardants, perfluorinated compounds, cadmium and hexavalent chromium. In addition, HBM4EU will address the issue of chemical mixtures; in this context, coexposure of consumers to mixtures of plant protection products will be addressed as a part of Group A.

\subsection{Review method}

The review was based on keyword research in three different scientific libraries: Web of science, Scopus and PubMed. The keywords, which were used referred to the classes of chemicals that are included in the $1^{\text {st }}$ set of priority compounds. Furthermore, generic PBTK models were included in the search criteria as they can simulate a wide range of chemicals during different life stages. The findings that corresponded to PBTK models for estimating ADME properties of several chemical compounds using directly human data or extrapolated data from animals to humans were included in the review. The studies that referred only to animal species and not to humans were excluded. For flame retardants, the only PBTK model that was identified was the one for BDE-47 in rats. Considering the lack of a human PBTK model, we decided (exceptionally) to include this model in the review.

Different combinations of keywords used are presented in the following table. 


\section{Human PBTK models identified for the priority compounds}

\subsection{Anilines}

Aniline is the prototypical aromatic amine and is predominantly used as a chemical intermediate for the dye, agricultural, polymer, and rubber industries (ATSDR, 2002). The general population may be exposed to aniline via inhalation through tobacco smoke, orally through diet or drinking water containing aniline in small amounts and rarely by living in uncontrolled hazardous waste sites. It has been reported that aniline causes methemoglobinemia (Harrison and Jollow, 1987; Kearney et al., 1984; Lee et al., 2013). Exposure to aniline can also provoke dizziness, headaches, irregular heartbeat, convulsions and coma.

One PBTK model that generated toxicity exposure distributions for a series of substituted anilines in rats and human was found in literature (Nguyen et al, 2015). The substituted anilines were chosen because of their structural similarity to aniline. This model consisted of two major sub-models that described disposition and input. In the disposition model, the relevant organs or tissues were selected. 14 compartments, including those for lungs, adipose, heart, brain, muscle, spleen, pancreas, stomach, gut, kidney, bone, skin, and thymus and two blood compartments (arterial and venous blood) were included. The input model was adapted from the advanced compartmental absorption and transit model (ACAT) (Agoram et al., 2001) originally developed by Yu et al (1999). It consisted of nine compartments, including stomach, large intestine, seven segments of small intestine, and corresponding enterocyte or GI wall. Each tissue was assumed to be perfusion-rate limited, as the compounds were reported to be rapidly absorbed from the small intestine, and passively permeated through tissue and cellular membranes with no known transporter. Finally, aniline was reported to be metabolised in liver by acetylation and hydroxylation pathways. The model was validated by comparing the whole blood concentration-time profiles for the five model compounds after an oral solution dose using the rat PBPK model. Different doses of aniline were simulated and compared with reported in vivo data of Harrison and Jollow (1987). The shape and the magnitude of predicted blood concentration-time course was in agreement with the observed data.

\subsection{Bisphenols}

The bisphenols are a group of chemical compounds with two hydroxyphenyl functionalities. Bisphenol A (BPA) is the most popular representative of this group and has been listed as endocrine disruptor by U.S. Environmental Protection Agency (EPA), as well as lately by ECHA (2018); although BPA was already in the REACH candidate list of substances of very high concern (SVHC), the entry was updated, to reflect its endocrine disrupting properties. BPA is used primarily as a monomer in the industrial production of epoxy resins and polycarbonate plastics (Keith, 1997). Thus, it is present in a wide range of consumer products, such as food and drink containers, as well as medical devices (EFSA, 2015; FDA, 2014; Willhite et al., 2008). There are several PBTK models in literature regarding human exposure to BPA.

The first model for BPA (for both rats and humans) was developed by Shin et al (2004a), where input parameters regarding human PBPK model were found using scaling methods from rat data. The compartments were divided into a) non-eliminating organs, which included spleen, 
kidneys, heart, testes, brain, muscle, adipose tissue, stomach and small intestine, b) an eliminating organ (liver), c) lungs, d) blood (vein and artery), e) lumen and f) small intestine. Differential mass balance equations were used for each individual compartment. For parameterisation and validation of the model, multiple intra-venous injections to rats and single intra-venous injection (5mg dose) and multiple oral administrations to steady state (100mg doses every $24 \mathrm{~h}$ ) to a $70 \mathrm{~kg}$ human were used. The same was also used for predicting time courses of BPA in blood and various human tissues. Observed concentrations of bisphenol $A$ in blood and other tissues, testis, brain, muscle, adipose tissue, stomach, and small intestine, were found after multiple iv injections to rats, together with the simulated individual time courses using the PBPK model. The average steady-state concentration of bisphenol A in all tissues except adipose tissue were higher than the corresponding blood levels. Good agreement was noted between predicted and observed concentration-time profiles for blood and all tissues despite the varying steady state concentrations.

Teeguarden et al. (2005a) developed a PBTK model for the evaluation of toxicokinetics of BPA for rats and human. Two sub models were established; one for BPA, consisting of the five compartments of gastrointestinal (GI) lumen, liver, blood, uterus and the remaining tissues and one for its metabolite glucuronide BPA (BPAG) including plasma and Gl lumen. Tissue distribution was represented as perfusion-limited in all the compartments, while no blood flow distribution was represented to the Gl lumen. This tissue was represented only as a compartment of absorption of BPA and of elimination and reabsorption of BPAG in the bile. Hepatic metabolism from BPA to BPAG was considered as second order kinetics. The metabolised BPA was transferred to the BPAG sub-model. A fraction of the formed BPAG was transferred to the Gl lumen and then was eliminated via the bile. The rest was transferred to the plasma compartment and was eliminated via urine. Another fraction of the BPAG eliminated to the GI lumen was hydrolysed to BPA and reabsorbed from the GI lumen to the liver at a first order rate. The extensive binding of BPA to plasma proteins in different species (including humans) in a percentage of $~ 90-95 \%$ of the total (Csanady et al., 2002; Kurebayashi et al., 2002) was in general comprised in the partition coefficient values from Csanady et al. (2002). Assessment of the model was carried out with BPA plasma and blood concentration data in rats, BPAG blood concentration and cumulative excretion of BPAG in urine and feces in rats, time course data of BPAG concentration in plasma in men and women after oral exposure to BPA as well as cumulative BPAG excretion in urine and feces in men and women. The error in the estimations was not explicitly quantified by the authors and the results were commented from graphic representations. The human assessment showed a good agreement of the simulated vs. the observed data from plasma at the beginning of the depuration $(<12 \mathrm{~h}$ postexposure) in men, while the data for women were scarce. An underestimation of the concentrations at later points of the time course, for both genders was noticed. A good agreement of the simulated vs. the observed excretion data in human was observed.

Based on the structure of the PBTK model with the BPA sub model coupled to a BPAG sub model, proposed by Teeguarden et al. (2005a), Edginton and Ritter (2009b) developed a model for predicting plasma concentration of BPA in children younger than 2 years old. The model included 15 organs as well as arterial, venous, and portal blood compartments. The organs were connected via blood flows, and the circulation system was closed via the lung. The oral absorption model was that of Willmann et al. (2004a) providing BPA input to the portal vein. Scaling the gastrointestinal parameters (gastrointestinal geometry, gastric emptying time, 
intestinal permeability, gastric and intestinal $\mathrm{pH}$, small intestinal transit time and intestinal surface area) to children between 0 and 2 years of age was also considered. The elimination of the total BPA was attributable to its metabolism to BPAG via glucuronidation by the enzyme UGT2B7. The intrinsic clearance of BPA to BPAG from adults to children was scaled. In general, it is noted that UGT2B7 activity in term neonates is only $5 \%$ that of adults, increases to $30 \%$ by 3 months of age and reaches adult levels by 1 year of age (Edginton et al., 2006a). It has to be noted that clearance was parameterised in a way that the observed blood time course of an adult fitted well the toxicokinetic profile of single BPA dose provided by Völkel et al. (2002).

Mielke and Gundert-Remy (2009) used a PBTK model allowing the simulation of blood concentrations of bisphenol $A$ at various life stages and the intake of bisphenol A as a discontinuous process thus mirroring the intake of the substance with meals. The basic model was similar to the model by Ramsey and Andersen (1984) but with some modifications for various life stages, including the newborn (Abraham et al., 2005). Excretion was modelled by metabolism in the liver to glucuronides and sulfate conjugated metabolites. Also this model was validated using the human voluntary data provided by Völkel et al. (2002). The predicted value of $2.6 \mathrm{ng} / \mathrm{ml}$ was somewhat higher than the experimental highest measured value of $2.3 \mathrm{ng} / \mathrm{ml}$, however it was considered as good, supporting the credibility of the model results. This model was further developed including the addition of skin absorption (Mielke et al., 2011), for the assessment of the contribution of dermal exposure to the internal exposure of bisphenol $A$ in man.

In addition, a PBTK model was developed by Yang et al. (2015) in order to assess the human exposure to BPA and its phase II conjugates BPAG and BPA sulfate (BPAS) in adult humans following oral ingestion. An eight-compartment PBTK model for BPA, including serum, liver, fat, gonads, richly perfused tissues, slowly perfused tissues, brain, and skin, and two singlecompartment (volume of distribution, $\mathrm{V}_{\text {body }}$ ) sub-models for BPAG and BPAS were constructed. According to the original model proposed by Fisher et al. (2011), the compartments were selected based on kinetic considerations (e.g. liver) and the model's potential use for internal dose metrics estimation and dermal exposure assessment (e.g. brain and gonads, skin). The uptake of BPA and BPAG in the tissues was described as a first order process. The phase II metabolites, BPAG and BPAS, were simply described as a single non-physiological compartment given that these conjugates display no known estrogenic activity. An In Vitro to In Vivo Extrapolation (IVIVE) approach was employed to derive model parameters representing hepatic glucuronidation and sulfation of BPA that were described using Michaelis-Menten equations. BPA glucuronidation occurred in the liver and the small intestine, while the formation of BPAS was assumed to occur only in the liver (Kurebayashi et al., 2010). Ten percent of BPAG derived from the small intestine and the liver was assumed to be secreted into the gut through the bile and undergo enterohepatic recirculation (EHR), whereas the remaining $90 \%$ of BPAG was taken up via the systemic circulation in the BPAG sub-model. The overall toxicokinetic behavior of the model was validated using the human volunteer data delivered by Völkel et al. (2002), who reported plasma concentration and urinary excretion-time profiles of $\mathrm{d}_{14}$-BPAG in adult humans after a single oral dosing of $5 \mathrm{mg} \mathrm{d}$-BPA over a period of up to 42 $\mathrm{h}$. These toxicokinetic data were used to optimise the parameterisation of key kinetic parameters. Simulations of cumulative excretion of total d6-BPA in urine were also in line with collected data, with MRD of $2.7(1.6-6.4)$ and AFE of $1.5(1.1-2.0)$. 
Lately, a generic PBTK model that captures satisfactorily life stage changes and physiological and metabolic efficiency change over an individual's lifetime (from conception till 80 years of age) was developed in INTEGRA European project and was used for the estimation of BPA toxicokinetics (Sarigiannis et al., 2016b). Furthermore, special attention was paid on the assessment of exposure to BPA during critical developmental stages such as gestation by modelling the mother-fetus toxicokinetic interaction. Arterial, venous and portal blood compartments, as well as all major human organs were included in the model. The main routes of exposure were described in detail and all the age and gender exposure modifiers were taken into account. To estimate the total amount of BPA to which the infant is orally exposed, the sum of free BPA and BPAG was considered, since all conjugated BPA is cleaved in the gastrointestinal tract. Three mass balance equations for a) red blood cells, b) plasma and interstitial tissue and c) cells were written regarding each tissue, allowing the application of the model to both flow limited and membrane-limited compounds. In this model, the parent compound (BPA) and the metabolite BPAG and BPAS (for fetus and early infancy) were taken into account, as the main detoxification pathway of BPA is phase II glucuronidation and sulfation at early developmental stages. Free BPA and BPAG were excreted through lactation, while BPAG was eliminated via urine for humans. This model was also validated with the human volunteer data from the Völkel et al. (2002) study for both BPA and BPA-glu in urine and the results fitted the experimental values with $R^{2}$ of 0.82 and 0.78 respectively.

Another model that accounted for in utero exposure has been developed by Gauderat et al. (2017). A feto-maternal TK model describing BPA and BPA glucuronide (BPAG) disposition in sheep was extrapolated to humans, while its parameterization was carried out using the human TK data obtained after d6-BPA administration on a cookie, to predict BPA and BPAG kinetics in the human mother-fetus unit. The model was able to capture fetal BPA internal dose under realistic maternal BPA dietary intake levels, with similar trough $(0.011 \mathrm{ng} / \mathrm{L}$ vs $0.014 \mathrm{ng} / \mathrm{L})$ and lower peak BPA concentrations $(0.023 \mathrm{ng} / \mathrm{L}$ vs $0.14 \mathrm{ng} / \mathrm{L})$ in fetal than in maternal plasma; the model-predicted BPAG concentrations in fetal plasma were consistent with reported levels in human cord blood that may be considered as relevant markers of the BPA dose entering blood throughout fetal life.

Finally, a model has been recently presented by Sharma et al. (2018b) that has taken stock from the advances and the parameterisation provided by the existing models. Metabolism in pregnancy was introduced via scaling of the in vitro Vmax for glucuronidation and sulfation, considering the pre-pregnancy body weight. The BPA metabolism data for the fetus was scaled using human in vitro data and fetus microsomal protein content, and, growing fetus liver and body weight. Two metabolic kinetic parameters namely $\mathrm{V}_{\max }$ (maximum rate of reaction) and $\mathrm{K}_{\mathrm{m}}$ (affinity of the substrate for the enzyme), for mother and fetus, were taken from in vitro studies and scaled to in vivo. Validation of the developed adult PBPK model was performed by comparing the model predictions with plasma data obtained from the human study by Thayer et al. (2015), in which volunteers were orally administered $100 \mu \mathrm{g} / \mathrm{kg} \mathrm{BW}$ dose of deuterated BPA. The observed concentration in different cohorts during pregnancy was used for P-PBPK model evaluation. For instance, maternal blood concentration during pregnancy or at the delivery time was used for exposure estimation. Five biomonitoring cohort data on fetus plasma, liver, mother placenta and amniotic fluid were used for model calibration and evaluation purpose. 


\subsection{Phthalates and DiNCH}

Phthalates is a huge class of chemicals used mainly as plasticizers in a wide variety of products, such as toys, vinyl flooring and wall covering, detergents, lubricating oils, food packaging, pharmaceuticals and personal care products (Heudorf et al., 2007; Kamrin, 2009). Humans are exposed through ingestion, inhalation, skin and eye contact. The primary route of exposure is through oral consumption mainly due to the transfer of substances from food packaging onto food stuff, as well as dust and soil ingestion (National Research Council, 2008). Phthalates have been reported to be responsible for several reproductive or developmental abnormalities (Meeker et al., 2009; Swan, 2008).

Clewell et al. (2008) developed a model for di-n-butyl phthalate (DBP) and its metabolites: monobutyl phthalate (MBP), MBP-glucuronide and oxidative metabolites of MBP in rat. Clewell et al. (2008) represented in detail the GI tract (upper, lower, wall), liver, plasma, certain reproductive tissues and either rest of the body or richly and slowly perfused tissues (depending on the sub-model), as well as feces and urine. The model of Gentry et al. (2011) for di(2ethylhexyl) phthalate (DEHP) and its metabolites in human was based on the model by Clewell et al. (2008). The main structural differences in the model of (Gentry et al., 2011) compared to the model by (Clewell et al., 2008) consisted in a simplified Gl lumen, non-application of the glucuronidation pathway and detailed representation of the oxidative-metabolites-submodel. In the models of (Clewell et al., 2008) and (Gentry et al., 2011), the distribution of the monoester was considered to be diffusion-limited, while the distribution of other compounds (diester, glucuronidated or oxidative metabolites) was represented as flow-limited. In both models, the diester was applied as a bolus to the Gl lumen. The metabolism of the diester was represented in the Gl compartment, liver and plasma. The monoester that arrives to the Gl lumen by metabolism of the diester (Clewell et al. 2008) or biliary excretion (Gentry et al. 2011) and the not metabolised diester were absorbed from the Gl compartment following $1^{\text {st }}$ order kinetics and transferred to the liver. The oxidation (MEHP, MBP) and glucuronidation (MBP) of the monoester were represented in liver. Clewell et al. (2008) included a hydrolysis of MBP-G to MBP in the Gl tract. A biliary excretion of the diester and the monoester in both models, as well as MBP-G (Clewell et al., 2008) and oxidative-MEHP (Gentry et al., 2011) was represented from the liver to GI lumen. The diester and all its metabolites were eliminated from the GI tract to feces. The monoester and oxidative monoesters in both models, as well as MBP-G in Clewell et al. (2008) were also eliminated from plasma to urine.

Later, a PBTK model was established as an exposure prediction tool for DEHP and DnBP in humans (Moreau et al., 2017), based on the model of Gentry et al. (2011), including the four interconnected sub-models for the diester, the hydrolytic monoester, the oxidative metabolites and the glucorinide conjugate. Diester distributed in the gut wall was passed to the liver via the portal blood, while their monoester metabolites MEHP and MBP were readily absorbed in the gut. Transport of monoester into the tissues was modeled using diffusion-limitation. Distribution of oxidative metabolites into the tissues was modeled using flow-limitation, assuming distribution with body water. DEHP is rapidly metabolised to its monoester, mono(2-ethylhexyl) phthalate (MEHP), which is further metabolised by various oxidation reactions to a number of secondary hydrolytic and oxidative metabolites that are conjugated via glucuronidation and other processes before being eliminated. DnBP is metabolised to its monoester, MBP. Oxidative metabolism was described in the liver using a saturable Michaelis-Menten description. A four- 
compartment model was used to describe different oxidative metabolites in the body. The model was validated for adult male rats using a dataset that is different from the one that was used to parameterise the model (Payan et al., 2001). No parameter values were tweaked to improve model fits to the "validation" data.

The recent model presented by Sharma et al. (2018b) is flow-limited and it comprises different compartments such as gut, liver, blood, fat and gonads. The metabolites of DEHP in liver and gut such as MEHP, 5-OH MEHP, 50xo-MEHP, 5cx MEPP and phthalic acid were described as a saturable process utilising Michaelis Menten kinetics. The in vitro intestinal and hepatic metabolic rates for different metabolites were reported by Choi et al., (2012) in human intestinal and hepatocyte cell line. Based on in-vitro studies of DEHP in the gut and liver, an IVIVE approach was implemented to derive model parameter such as Vmax, describing the maximum rate of metabolism. The estimated parameters $V_{\max }$ (in-vitro), and maximum rate of reaction were scaled to the whole body.

\subsection{PAHs}

Polycyclic Aromatic Hydrocarbons (PAHs) are a group of chemicals that are formed during the incomplete burning of coal, oil, gas, wood, garbage or other organic substances, such as tobacco. PAHs generally occur as complex mixtures and not as single compounds. A few PAHs are used in medicine and to make dyes, plastics and pesticides. Others can be contained in substances such as crude oil, coal, coal tar pitch, creosote, and roofing tar. The primary sources of exposure to PAHs for the general population are via inhalation through tobacco smoke, wood smoke and ambient air, as well as via food ingestion. The majority of people are exposed to PAHs in the workplace. PAHs have also been found in other facilities where petroleum, petroleum products, or coal are used or where wood, cellulose, corn or oil are burned. People living near waste sites containing PAHs may be exposed through contact with contaminated air, water and soil (ATSDR, 1995).

Health effects from chronic or long-term exposure to PAHs may include decreased immune function, cataracts, kidney and liver damage, asthma-like symptoms and lung function abnormalities. Mixtures of PAHs are considered carcinogenic to humans (ATSDR, 1995; World Health Organization, 2010). The toxicokinetic profiles of benzo(a)pyrene (BaP), which has been determined by EPA (2013) as possible human carcinogen, and its metabolite 3hydroxybenzo(a)pyrene (3-OH-BaP) were investigated by a PBTK model found in literature (Heredia-Ortiz et al., 2014). Two sub-models for $\mathrm{BaP}$ and 3-OH-BaP were constructed, while other possible metabolites were represented as compartments in these two sub-models. Eleven compartments of lungs, AT, skin, kidney, liver, Gl tract, "rest of the body", arterial blood, venous blood, feces, "other metabolites" were developed for BaP. The same compartments adding bladder and urine were considered for the metabolite 3-OH-BaP. Oral, dermal exposure and exposure via inhalation was taken into account. It was determined whether the distribution in each organ was limited by tissue perfusion or diffusion (Heredia-Ortiz and Bouchard, 2013). Hepatic metabolism and elimination were described as first-order processes. PBPK model simulations were compared to predictions obtained with a simple compartmental toxicokinetic model. Both models provided similar fits to the observed data. For most workers, a more pronounced difference was observed between simulated and observed time courses when considering an exposure by inhalation. Furthermore, the inhaled dose scenarios simulated to 
reproduce the urinary profiles were in most cases higher than those recorded from airborne measurements of BaP concentrations in the facilities and time-of-shifts. Cases in which the simulated inhaled BaP concentration levels needed to obtain a better fit to observed profiles were much higher than measured air concentration values further indicate that inhalation was not the main route of exposure. Good agreement of the simulated vs. the observed data was obtained when considering only the dermal exposure route. Simulations thus suggest a mostly dermal exposure of BaP during work.

The PBTK model for BaP exposure via inhalation in rats and humans (Campbell et al., 2016) is an extension of a previously published oral BaP model for rats (Crowell et al., 2011). In Crowell's model, lung, liver, fat and richly and slowly perfused tissues, as well as oral bolus, duodenal and intravenous (IV) infusion exposure routes were modeled. Oral exposures were represented as a two-compartment Gl tract, while BaP was metabolised to total metabolites in the lung and liver. Fecal elimination of $\mathrm{BaP}$ was also included. BaP distribution from blood to tissues was assumed to be blood-flow limited except for the fat compartment, which was diffusion-limited. The current model was expanded in order to include a description for the $3-\mathrm{OH}$ $\mathrm{BaP}$ metabolite. Elimination of free 3-OH BaP from plasma was described as two separate clearances with one representing urinary clearance and the other accounting for a lumped metabolic/fecal clearance. The model-predicted pooled creatinine corrected urinary $3-\mathrm{OH} \mathrm{BaP}$ was compared with measured concentrations found in literature (Lafontaine et al., 2006; 2004). The model prediction ranged within a factor between 2 and 3 . It has also to be noted, that the particle size distribution induces uncertainty related to the actual uptake of PAHs through inhalation; it has been found that PAHs uptake depends not only on the amount that is adsorbed on airborne particles, but also on the particle size distribution, which in turn affects the particles retained (and the respective PAHs) across the human respiratory tract (Sarigiannis et al, 2016). However, the model captured successfully the relationship between exposure and urinary excretion of $3-\mathrm{OH} \mathrm{BaP}$ for a broad range of $\mathrm{BaP}$ exposure levels.

\subsection{Brominated flame retardants}

Brominated Flame Retardants (BFRs) belong to a large group of substances known as organohalogens that tend to reduce the flammability of products and are used in plastics, electronics, clothes and furniture to prevent fire accidents. Some of the BFRs accumulate via the food chain and the environment and they have been characterised as persistent and toxic as it is suspected to cause endocrine disruption and neurobehavioral effects (Fromme et al. 2016).

Emond et al. (2010) developed a PBPK model for exposure assessment of BDE-47, one of the most toxic flame retardants, in adult female pregnant and male rats. The PBPK model included the main compartments of brain, liver, adipose, kidney, placenta, fetus, blood, while the rest of the organs were represented by one compartment. The placenta and the fetus were only activated for the pregnant female rats. Most of the compartments were represented as perfusion-limited. However, liver, adipose, brain and fetus were represented as diffusion-limited. BDE-47 was metabolised via liver and eliminated through urine and feces. 


\subsection{Pesticides (chlorpyrifos)}

Pesticides are chemical compounds used in agriculture to kill pests and in public health to kill vectors of disease, such as mosquitoes (Nicolopoulou-Stamati et al., 2016). By their nature, pesticides are potentially toxic to other organisms, including humans, and need to be disposed properly. Agricultural and public health workers are greatly exposed to hazardous pesticides during their application. Exposure is mainly by dermal route for preparation of sprays and by dermal and inhalation routes during application. Ingestion might occur through consumption of contaminated food during or following work or through oral contact with contaminated hands. Exposure of the general population to pesticides is lower and occurs mainly through consumption of residues of pesticides in food and drinking water (WHO, 2010). Acute health problems that are linked with pesticides are abdominal pain, dizziness, headaches, nausea, vomiting, as well as skin and eye problems (Klaassen, 2007). Associations have been found with different types of cancer and health effects in nervous system, cardiovascular system, gastrointestinal tract, liver, kidneys, reproductive system, endocrine system and blood (Gilden et al., 2010; Sanborn et al., 2007).

There are few PBTK models found in literature, mainly developed for chlorpyrifos (CPF), one of the main pesticides used on crops, animals and buildings. A PBTK model was developed to describe the time course of ADME properties of CPF and its metabolites, CPF-oxon and 3,5,6trichloropyridinol (TCP) in rat and human (Timchalk et al., 2002). The model assumed that the toxicokinetic response in rats and humans was independent of gender. The absorption of CPF required the use of a two-compartment uptake model. This two-compartment model incorporated 1st order rate equations to describe systemic uptake and transfer between compartments. In addition, absorption of CPF from the diet was incorporated into the model to allow the simulation of chronic dietary administration. TCP was formed by direct CYP450 metabolic conversion of CPF and through A-EST-mediated hydrolysis of CPF-oxon and B-EST binding of CPF-oxon, respectively. The CYP450 activation/detoxification and A-EST detoxification of CPF-oxon were limited to the liver compartment and were all described as Michaelis-Menten processes. The blood kinetics and urinary elimination of TCP were described with a single, one-compartment model utilising a 1st order rate of urinary elimination. The model was evaluated against the experimental data of Yano et al. (2000), that were found to be consistent with the model simulations. However, some quantitative differences revealed that eventually other metabolic pathways might be activated at higher exposure levels.

Another PBTK model (Lu et al., 2010) was developed based on the PBTK model for CPF in rats and humans (Timchalk et al., 2002). Metabolism of CPF was assigned to occur in the blood and liver, with the excreted metabolite, TCP, lumped into a single compartment. The metabolite description was expanded from a volume-of-distribution concept to a physiologic description that can incorporate known differences at different life stages, such as between children and adults. Three exposure scenarios were developed, including a) bolus ingestion (based on levels measured in three meals throughout the sampling day), b) inhalation and c) rate ingestion for non-dietary routes (such as hand-to-mouth activity), while the dermal route of exposure was not considered. The model was evaluated using aggregate exposure data against cumulative urinary excretion data. The model predictions, regarding the major metabolite TCPY in urine, were compared with measured data obtained from children (Nolan et al., 1984; Timchalk et al., 
2002). The calculated levels were consistent for some of the children, but in principle it seemed to underestimate TCPY excretion in urine.

\subsection{Perfluorinated compounds}

Poly- and perfluoroalkyl substances (PFASs) are a class of man-made chemicals that contain a fluorinated alkyl chain and a non-fluorinated group consisting of oxygen, hydrogen, sulfur and/or nitrogen atoms. PFASs have widely been used in consumer and industrial applications, including protective coatings for fabrics and carpets, paper coatings, insecticides, paints, cosmetics and fire-fighting foams (Domingo, 2012, Wang et al. 2014). It has been shown by several studies in humans that certain PFAS may affect the developing fetus and child, including possible behavioral and developmental problems, it may decrease fertility and interfere with the body's natural hormones, increase cholesterol, affect the immune system, and increase cancer risk (ATSDR, 2017).

Perfluorooctanoic acid (PFOA) and perfluorooctane sulfonate (PFOS) are the most widely spread and studied PFASs. Toxicokinetic characteristics of PFOA and PFOS have been studied by Loccisano et al. (2011) in monkeys and humans. The model consisted of nine compartments, including plasma, gut, liver, fat, skin, kidney, "rest of body", filtrate and urine storage. Plasma was represented instead of blood because these compounds do not partition into the red blood cells. The distribution into different tissues was considered as perfusion-limited and no metabolism of the compounds was assumed as it is reported that PFOS and PFOA are well absorbed orally, poorly eliminated and not metabolised (Johnson et al., 1984; Vanden Heuvel et al., 1991). The PFOA model for humans was assessed by comparing plasma and serum data from populations exposed by contaminated water supplies. The concentrations measured in water by the same or other studies were used to simulate the exposure in the model. The two tested reported half-lives let a better fitting of simulated vs. observed data for the data during exposure than during depuration. For both PFOA and PFOS, the assessment process included the comparison with serum data from general population, before and after the phase-out of these chemicals. The results indicated good agreement of simulated vs. experimental data. After validation, the model was used to estimate the half-life, from individual data on PFOA from one study. The estimated individual half-lives were in the range of those estimated by Olsen et al. (2007) and Bartell et al. (2010).

Loccisano et al. (2013) modified a previously developed rat model (Loccisano et al., 2012) in order to be extended to the gestation and lactation life stages and provide estimates of maternal, fetal, and infant exposure to PFOA and PFOS for adult humans. The structural changes made were the addition of mammary tissue, placenta, fetal compartments and a milk compartment for lactation. Only the mother was exposed directly, maternal exposure was by direct intake and through drinking water and infant exposure was through milk. Fetal exposure to PFAA was through placental transfer, which was described as a bidirectional transfer process between free chemical in the placenta and fetal plasma. Placental transfer was described by a simple diffusion process, while transfer from the plasma to the milk compartment was assumed to be flow-limited. Only the free fraction of chemical in plasma was available for uptake into tissues or milk. Clearance of PFAA in the mother was supposed to occur by urinary elimination from the filtrate compartment in both models; clearance in the infant was described with a 
generic first-order rate constant from the central compartment. To validate the model, multiple human data related to pregnancy or lactation were used. With regard to the lactation model, if a plasma concentration was reported, the exposure was estimated to yield this concentration and then the resulting predicted milk or infant blood concentration was compared to the observed value to evaluate the model structure and parameters. The validity of the model was evaluated by comparing the predicted with the measured values, using graphical methods. Exposure levels were practically estimated using the try and error method.

A new PBTK model was developed by Fabrega et al. (2014) for PFOA and PFOS based on the previously described one (Loccisano et al., 2013). The key process adopted in the model was the kinetics of resorption by renal transporters in the filtrate compartment, where chemicals were reabsorbed back to plasma through a saturable process. In addition to plasma, gut, liver, fat, kidney, filtrate and the remaining body compartments, the adapted PBTK model included lungs and brain. However, skin was removed as it is not a potential site of absorption/accumulation for PFASs. Human exposure to PFOA and PFOS was evaluated through water consumption and food intake, which was found to be the most important contributive route to the exposure of PFOS and PFOA. Gut was selected as an absorption site, brain as target organ of PFASs neurotoxic effects, liver as an accumulative tissue for organic chemicals, lungs as they may exhibit immaturity after PFOS exposure, fat as a main site of accumulation in lipophilic tissues and kidney for its role in elimination. For model validation, the computational results were compared with data reported in human tissues (Ericson et al., 2007; Pérez et al., 2013). Although the model had been successfully validated by using experimental data in human blood, for other human tissues the results were not very satisfactory.

\subsection{Metals}

\subsubsection{Hexavalent chromium}

Chromium is an element that can be found in rocks, animals, plants and soil. It is widely used in manufacturing processes and can be found in many consumer products, such as wood treated with copper dichromate, leather tanned with chromic sulfate and stainless-steel cookware (ATSDR, 2012). High concentrations of chromium VI in environmental media (air, soil and water) can be generally attributed to industrial releases, use and disposal of chromium-based products. However, low concentrations of $\mathrm{Cr}(\mathrm{VI})$ have been reported in urban household dust (Stern, 2010), in some foods (Mandiwana et al., 2011) and in drinking water due to Cr-enriched geology (Mills et al., 2011; Oze et al., 2007). Health problems resulting from chronic exposure to chromium are related to the respiratory tract, including airway irritation, airway obstruction, as well as asthma. Condition of very high inhalation exposure to chromium can exert a range of toxicities, including lung, nasal, or sinus cancer (ATSDR, 2011; Langard, 1990; O'Flaherty, 1995).

Several physiologically based pharmacokinetic models have been developed to describe the behavior of $\mathrm{Cr}(\mathrm{VI})$ in humans. One of the developed PBTK models (O'Flaherty et al., 2001) was based on existing simpler models for human (O'Flaherty, 1993) and rat (O'Flaherty, 1996). The adaptations included differential absorption of $\mathrm{Cr}(\mathrm{VI})$ and $\mathrm{Cr}(\mathrm{III})$, rapid reduction of $\mathrm{Cr}(\mathrm{VI})$ and $\mathrm{Cr}$ (III) in all body tissues and fluids, modest incorporation of chromium into bone and concentration dependent urinary clearance. The model did not include uptake through the skin and a lung compartment. However, it could estimate the upper limit on pulmonary absorption of 
inhaled chromium. In this model, oral exposure to $\mathrm{Cr}(\mathrm{VI})$ and $\mathrm{Cr}(\mathrm{III})$ salts via diet and drinking water was assumed. Due to the rapid appearance of $\mathrm{Cr}(\mathrm{III})$ and $\mathrm{Cr}(\mathrm{VI})$ in the blood, both were considered to be absorbed from the stomach and intestine. Once in the blood, $\mathrm{Cr}(\mathrm{VI})$ was assumed to be rapidly reduced by glutathione and hemoglobin to $\mathrm{Cr}(\mathrm{III})$. Chromium has been observed to be excreted in the bile and across the wall of the gastrointestinal tract. The rapidity of reduction of $\mathrm{Cr}(\mathrm{VI})$ to $\mathrm{Cr}(\mathrm{III})$ suggests that there is not urinary excretion of $\mathrm{Cr}(\mathrm{VI})$ (Minoia and Cavalleri, 1988). However, a pathway for urinary excretion of $\mathrm{Cr}(\mathrm{VI})$ was included in the model. The time course of chromium in plasma, as well as the excretion rate in urine were evaluated using HBM data from a published volunteer study (Paustenbach et al., 1996). In this study, a volunteer received multiple doses ( 5 within a day) of potassium dichromate $\left(\mathrm{K}_{2} \mathrm{Cr}_{2} \mathrm{O}_{7}\right)$, amounting for a daily intake of $4.0 \mathrm{mg} \mathrm{Cr}(\mathrm{VI})$, for 17 days in the row. Multiple blood and urine samples were collected before, during and after (for another two weeks) the cessation of the experiment. Despite the highly variable exposure scenario, as well as the wide intra-day variability of the urinary excretion rates, the simulated data were able to trace successfully the diurnal variability of both measured blood and urinary data.

Another human PBTK model was adapted from the rodent PBTK model proposed by Kirman et al. (2012) to describe the toxicokinetic processes of $\mathrm{Cr}(\mathrm{VI})$ and $\mathrm{Cr}(\mathrm{III})$. Gastrointestinal lumen, oral mucosa, stomach, small intestinal tissue, blood, liver, kidney, bone and a combined compartment for remaining tissues were included in the model. For human, the following changes were made: a) multiple bolus exposure events per day were represented, to accommodate the exposure regimens implemented by some human studies; b) two input parameters were added to permit scaling of systemic tissue uptake and release rate constants from mice; c) absorption was modeled in humans as a first order process; d) compartments for duodenum, jejunum, and ileum were lumped into a single compartment for the small intestines and e) the oral cavity compartment was removed from the model since there was no human data available for the oral cavity. Chromium absorption was expected to occur primarily within the small intestine, while $\mathrm{Cr}(\mathrm{VI})$ that reached portal plasma was subject to several competing processes: a) reduction to $\mathrm{Cr}(\mathrm{III})$; b) uptake into erythrocytes; and c) transit to the liver and systemic tissues. The reduction of $\mathrm{Cr}(\mathrm{VI})$ was described as a $\mathrm{pH}$-dependent process. Within the $\mathrm{Gl}$ tissue, $\mathrm{Cr}(\mathrm{VI})$ was subject to further reduction. The majority of chromium in the Gl lumen remained unabsorbed and was excreted in feces, while biliary excretion of chromium was considered to be negligible. To validate the model, the data sets of Finley et al. (1997a) were used. The predictions of the model fitted well plasma levels related to 2 different doses, of 5 and $10 \mathrm{mg} \mathrm{Cr}$ respectively. However, the model was not able to sufficiently distinguish the lower exposure levels (below $1.0 \mathrm{mg} \mathrm{Cr}$ ) examined by Finley et al. (1997a). Beyond that, the model described chromium toxicokinetics sufficiently for typical exposure scenarios.

\subsubsection{Cadmium}

Industrial and agricultural activities are the main sources of cadmium (Cd) release in the environment (Nordberg et al., 2014). Cadmium is a toxic metal, which can mainly enter the human body either orally via food intake (shellfish, liver and kidney meat, cereal products, leafy vegetables) or via inhalation (cigarette smoke). It is considered as one of the metals that poses great threat to human health, as it is associated with a broad spectrum of health conditions, including cardiovascular, kidney and bone diseases (Jarup and Akesson, 2009; Nordberg et al., 2014; Satarug et al., 2010), as well as breast cancer and neurotoxic effects (Nordberg et al., 2014). 
Some of the PBTK models developed for human exposure to metals are presented below. The first model for cadmium uptake and retention was proposed by Kjellström and Nordberg (1978), taking into account the exogenous body compartments of lung, intestines and kidney and the endogenous compartments of other tissues. Two cases of cadmium exposure through inhalation as particulate matter were included: smoking and work in a battery factory. After inhalation, cadmium was deposited in the nasopharyngeal, the tracheobronchial, and/or the pulmonary compartments in different proportions, depending on particle size and respiratory characteristics, and then transported by mucociliary clearance to the pharynx. Cadmium distribution in the liver and/or kidney was dependent on the proportion of plasma cadmium bound to metallothionein protein. Cadmium distribution in the body depending on the absorption by alveolar capillary or portal blood was not known and therefore not implemented. Regarding the elimination process, a certain amount of cadmium in blood was assumed to be excreted via intestine to the feces, while an amount of cadmium in liver was assumed to be excreted via bile to the feces. The calculated cadmium concentrations were validated using data from Elinder et al. (1976) and Kjellström et al. (1977). Overall, the model presented difficulties in addressing high levels of exposure that were mainly attributed to specific assumptions regarding urinary excretion.

Another PBTK for Cd was developed (Ruiz et al., 2010a), based on the first model but with some modifications regarding the inter-compartmental transfer of $\mathrm{Cd}$, the growth algorithms for males and females and corresponding organ weights for the estimation of age-specific Cd concentrations. The model simulated lung, liver, kidney, three blood compartments (plasma, erythrocytes and metallothionein) and other tissues and considered two routes of exposure; inhalation and oral. For inhalation exposure, the model accounted for different deposition patterns for different size particles in different regions of the respiratory tract, while for the oral exposure, Cd may enter the GI tract via food and water or as Cd particles embedded in mucus from the respiratory tract via the mucociliary escalator. The model was validated both against a) simulations to other published model simulations and b) to measured biormarker data (CDC, 2009). The model predicts the latest NHANES data well. However, the range of results showed a better correlation for the males than for females. The model predicted urinary $\mathrm{Cd}$ concentration for males (non-smoking) aged 6-59 years, but some predictions were towards the upper limit of the range found in the actual survey. The model over-predicted the actual values for females (non-smoking) aged 6-59 years. This may be due either to potentially outdated intake estimates from NHANES III 1988-1994, or due to the slower elimination of cadmium for females, that was not properly accounted for in model parameterisation.

The model proposed by Kjellström et al. (1978) was also used and modified by Bechaux et al. (2014). In order to take the impact of gender into account, triangular distributions instead of single input values, the ranges of them provided by literature (Ruiz et al., 2010a). Bayesian population model was applied to link external exposure to internal dose provided by the urinary concentrations data. For validation purposes, computationally predicted urinary concentrations were compared with the measured ones. This method was proposed for the interpretation of the National Health and Nutrition Examination Surveys (NHANES) data (Ruiz et al., 2010b). The results showed a significant underestimation of cadmium concentrations in urine predicted by the model. As proposed in the discussion of the work by Ruiz et al. (2010b), due to the long half-life of cadmium in the human body and its accumulation in kidneys, a potential trend in exposure can explain an underestimation of current urinary concentration. 


\section{Generic PBTK models}

Generic PBTK models, are toxicokinetic models that account for the main human anatomy (i.e. all major tissues and body compartments) and physiology (i.e. all major processes related to absorption, distribution, metabolism and elimination), independently of the compound for which they describe its toxicokinetic properties. This well-defined compartmental structure is much closer to real-life physiology and the applicability of the model is ensured by the compoundspecific parameterisation. In contrast, compound specific PBTK models focus on the compartments and the processes that are essential to capture the toxicokinetic properties of the specific compound(s). As a result, compound specific PBTK models may differ significantly in the structure and the processes that are included in the conceptual model. The latest years, the interest for generic toxicokinetic models is continuously growing, since they are able to provide rapid screening-level approach to PBTK modeling. In our view, generic PBTK provide a high level of transparency and flexibility as they can predict the ADME properties of a wide range of chemical compounds during the different stages of human life. One of the developed generic PBTK models is the INTEGRA model, which covers a broad range of compounds from different chemical families (Andra et al., 2015; Sarigiannis et al., 2014; Sarigiannis et al., 2016). These include BPA, benzene, toluene, ethylbenzene, xylene, trichloromethane, phthalates, PAHs, Polychlorinated Dibenzo-p-Dioxins (PCDDs), Brominated Diphenyl Ethers (BDEs), pesticides, parabens and perfluorinated compounds.

The generic human PBTK model developed in INTEGRA was designed to cover major ADME processes occurring in the human body at different life stages, to be easily applicable to a broad variety of chemicals after compound specific parameterisation. In practice, it accounted for mother-fetus interaction, capturing all age dependent physiological changes, including the ones of the pregnant mother. All major human organs were included, as well as arterial, venous and portal blood compartments. Xenobiotics and their metabolites were linked through the metabolising tissues.

The model in its generic form included the parent compound and up to three generations of potential metabolites. The mass balance equation for each compartment described all processes with biological significance, such as absorption, metabolism, elimination, and protein binding. In practice, in each tissue three mass balance equations were written, for a) red blood cells, b) plasma and interstitial tissue and c) cells. Specific organs were further divided in subcompartments: liver was divided in up to 5 compartments so as to better describe the distribution of enzymes and brain was divided in four sub-compartments, namely, main brain, globus palidus, cerebellum and pituitary, so as to better describe the permeability differences among the different brain regions.

The model described mother-fetus interactions by modelling the intra-placental properties that governs the transfer of xenobiotics and their metabolites from the mother to the fetus as it grows. The anthropometric parameters of the models were time dependent in order to provide a lifetime internal dose assessment, as well as to describe the continuously changing physiology of the mother and the developing fetus.

The model included the diffusion flow from uterus to placenta and vice-versa during pregnancy (Beaudouin et al., 2010). Excretion via lactation was described as an output from the mammary tissue compartment through a partitioning process between mammary tissue and milk, and milk 
withdrawal by suckling, as described for PCBs in rats (Lee et al., 2007) and further adopted for humans (Verner et al., 2008).

The model included also a detailed description of the three main routes of exposure. Inhalation took into account absorption of gases and deposition fractions of particles across the different human respiratory tract regions based on particles size distribution. Absorption through the oral route was governed by the absorption rates of stomach and intestine. To better describe dermal absorption, skin was modelled as a two layer structure, including stratum corneum that has been described as a "bricks and mortar" structure (Touitou, 2002) and viable epidermis (also accounting for metabolism), where the geometry of all layer microstructure has been explicitly described (Mitragotri et al., 2011). The fact that in each tissue three mass balance equations were written for a) red blood cells, b) plasma and interstitial tissue and c) cells, allowed the application of the model to both flow limited, as well as membrane-limited compounds, by just changing the parameterisation of the membrane permeability product. Although the main metabolic organ was the liver, also other sites of metabolism could be considered (intestine, brain, skin, placenta) based on the presence or not of the enzymes involved in the metabolism of the compound of interest.

Metabolism was described by saturable kinetics. Elimination was described by urine and feces excretion, as well as through lactation for females (when applicable). Binding to red blood cells and plasma proteins were described by the equilibrium of concentration between red blood cells and plasma (accounting also for the hematocrit levels), as well as the fraction unbound in plasma.

For the compound specific parameterisation of the model, data was obtained (a) either from existing experimental values for previous compound-specific PBTK models, or (b) by using advance QSAR models (Sarigiannis et al. 2017; Papadaki et al. 2017) developed in the frame of INTEGRA for tissue:blood partition coefficients and biochemical parameters such as Michaelis Menten constant and Maximal Velocity. This strategy resulted in the parameterisation of totally 136 chemical compounds, for which more than 110 compounds parameterisation was done (or completed) using the above QSAR models.

Jongeneelen and Berge (2011) designed the IndusChemFate generic model primarily for neutral and predominantly ionic organic compounds. The model contained eleven body compartments (lung, heart, brain, skin, adipose, muscles, bone, bone marrow, stomach and intestines (lumped), liver and kidney) and assumed a reference human of $70 \mathrm{~kg}$. Tissue concentrations for each of the chemicals and metabolites could be simulated for either acute, occupational or environmental exposure regimes with its typical duration, routes, concentrations or dose rate. Inhalation in the PBTK model was controlled by the concentration of the compound in the inhaled air, the alveolar ventilation, and the blood/air partition coefficient. For dermal absorption, a modified version of the algorithm developed by ten Berge (2009) was applied.

The physiological model considered the following processes: 1) dermal deposition of a substance on the skin, 2) diffusion to the stratum corneum and 3) absorption to the dermis/blood flow. Oral intake of compounds was considered as a bolus applied to the intestinal lumen (via the stomach) and then absorbed into the intestinal tissue at a first order rate. From the intestines, the compound was released to the blood stream via the liver. Stomach and intestines were lumped in the model. 
This model incorporated enterohepatic circulation by defining the ratio of excretion to bile relative to excretion to blood. The chemical in the human body was eliminated in the model by metabolism and direct excretion in air or urine. Metabolism was described by Michaelis-Menten saturable metabolism following the mathematical algorithms as described by Ramsey and Andersen (1984). The parent compound was metabolised by a set of (iso)-enzymes. Usually, one or more metabolites were produced, which may either undergo further metabolism or be excreted. Contrary to many PBPK models, the occurrence of metabolism was not limited to the liver compartment but could be considered in any of the 11 model compartments. However, the default setting was metabolism in the liver only. Compounds could be excreted via urine, either unchanged as parent compound or as a metabolite. The model took into account the renal clearance of substances by means of ultrafiltration in the glomeruli and possible resorption to the blood in the tubuli. When the volatility is high, chemicals are exhaled.

The generalised PBPK model of MENTOR-3P (Georgopoulos and Lioy, 2006) was adopted and simplified as "flow-limited" PBPK formulation in order to capture the dynamics of four As circulating species in body compartments (arsenates, arsenites, and the As metabolites MMA and DMA) (Xue et al., 2010). Dietary and drinking water exposure was considered.

Furthermore, a stochastic PBTK model was developed by Beaudouin et al. (2010) that has been tested on 1,3-butadiene (BD) and 2,3,7,8-tetrachlorodibenzo-p-dioxin (TCDD). This model included 22 detailed compartments of the human body. All tissue compartments were assumed to be well-mixed and blood flow-limited. The lungs were separated into two compartments to distinguish the pulmonary functions from the lungs anatomy. Nineteen compartments of the placenta, the amniotic fluid and fetus organs were added to the general PBPK model to describe pregnancy and fetal development. Inhalation and ingestion were integrated in the model. The gastrointestinal tract was subdivided into the stomach and the guts. Each of these was divided into 2 compartments: the lumen and the wall. The exchanges between the lumen and the wall were modeled as a diffusion by a first order reaction. The distribution was assumed to be homogeneous throughout the compartment volume and to be limited by perfusion, i.e., the tissue membranes present no barrier to diffusion. Elimination occured via metabolism, urinary and faecal excretion, exhalation and, in case of lactation, milk production. Liver, lungs, gut and placenta represented the four sites of metabolism.

The MERLIN-Expo tool (Brochot and Quindroit, 2018) was applied to inorganic chemicals (e.g. lead and arsenic), perfluorinated compounds (PFOA, PFOS) and persistent organic pollutants (PCBs, dioxins). The 'Human' model implemented in MERLIN-Expo was a PBTK model that subdivided the body in 22 compartments and parameterised with relationships describing the time evolution of the physiology and anatomy of the individuals. The PBTK model accounted for the physiological or biochemical variations that arise throughout the growth and the development of an individual. This model was based on the model developed by Beaudouin et al. (2010) and has been improved to include new features (e.g., binding). Absorption of the contaminants via inhalation was considered. Gas exchanges (inhalation and exhalation of contaminant, oxygenation of blood) in the alveolar space were assumed to be very rapid. The alveolar space was located between the venous blood and the lungs and modeled as an organ. A simple model describes gas exchanges based on a one-directional airflow in the region of gas exchange and a rapid equilibrium between lung air and blood in the alveoli. Absorption by ingestion was also taken into account. The gastrointestinal tract was subdivided into stomach and guts. Each of these was divided into the lumen and the wall, in which the exchanges 
between the lumen and the wall were modeled as a diffusion by a first order reaction dependent of the concentration of contaminant in the stomach or gut lumen. Alternatively, a direct input could be made in the liver. Distribution refers to the reversible partitioning of a compound into the various tissues of the body from the systemic circulation. Each organ or tissue could receive different doses of the compound and the compound can remain in the organs or tissues for a varying amount of time. The distribution was assumed to be homogeneous throughout the compartment volume and limited by perfusion, i.e., the tissue membranes present no barrier to diffusion. In this PBPK model, metabolism could occur in all compartments except in gut and stomach lumen and in alveolar space. Two equations were proposed to model metabolism either as saturable (Michaelis-Menten equation) or linear process (first order reaction). Excretion could occur in all organs/compartments except in gut and stomach lumen and in alveolar space and was described by a first-order reaction. The biliary excretion and the excretion in feces were considered as the two specific excretion routes in the model. Contaminants excreted by bile entered in the gut lumen and could be reabsorbed. Finally, model predictability is evaluated via direct comparison between computational predictions and experimental data on small case studies.

\section{Discussion}

A comprehensive review of the available human PBTK models for the 1st set of priority compounds is presented herein. With regard to the specific chemical families and compounds examined, large discrepancies regarding the availability of models were observed. For compounds like BPA and cadmium, there are several available models, while for compounds like brominated flame retardants there is a paucity of human PBTK models. Moreover, it has to be highlighted that there is limited availability of models for the various compounds relevant for the chemical families that eventually include many compounds, e.g., althouth there are many models for BPA, and there is no model for the alternatives BPS and BPF, while similar is the situation for the rest of the substance groups.

Regarding BPA metabolism, all models accounted for a very rapid metabolism, including the first models developed by Shin et al. (2004a) and Teeguarden et al. (2005a). The significant differences between neonates and adults were clearly illustrated and described with metabolic scaling by Edginton and Ritter (2009b). The core finding of their study was that BPA plasma concentrations could be approximately eleven times greater in newborns than in adults exposed to the same weight-normalised dose. In the BPA model developed by Mielke and GundertRemy (2009), sulfation was also included as an additional metabolic pathway, assuming that sulfation activity is well expressed in newborns and it is at least as high as in adults, or even higher. Finally, based on the physiologically-based approach for scaling to children (Edginton et al., 2006a) and the more recent findings regarding the ontogeny of enzymes involved in BPA detoxification (Court et al., 2012; Leeder, 2009), in the most recent BPA model by Sarigiannis et al. (2016b) clearance rates were adjusted taking into account the findings of Fisher et al. (2011) and assuming an age-dependent bioavailability difference factor of 2 .

Moreover, the model of Sarigiannis et al. (2016b) was used for reconstructing exposure from EU-wide HBM data, while its explicit description of mother-fetus interaction allowed the estimation of the effect of placental deconjugation on fetus internal exposure. Finally, the model 
presented by Sharma et al. (2018a) evaluated the most recent kinetic data from control human experimental study and extended the adult model to the pregnancy PBTK model with the available BPA biomonitoring cohort studies. The prediction of higher concentration of BPA during the mid-gestational period in the amniotic fluid, placenta, and the fetus liver was in accordance with biomonitoring data, indicating that mid-gestational period might be the critical window of exposure for the fetus. Similar findings regarding in utero exposure to BPA have also been reported by Gauderat et al. (2017), although the specific model included a smaller number of compartments, reflecting a hybrid between PBTK and TK models.

With regard to metals, three human PBTK models were identified for cadmium and two for hexavalent chromium. With regard to cadmium, most of the models are able to capture the complex interactions of cadmium with biological systems. However, it has to be highlighted that among the different models, the one of Bechaux et al (2014) is able to capture the close relation between cadmium absorption and the expression of the divalent metal transporter 1 (DMT-1), which transports cadmium and iron into the mucosa cell in a competitive manner, by using a different absorption factor for male and female in the general population. Moreover, this situation seems to be exacerbated during pregnancy when enterocytes have an increased DMT-1 density at the apical surface to optimise micronutrients absorption, affecting the overall biologically effective dose. With regard to hexavalent chromium models, the model presented by O'Flaherty et al. (2001) includes differential absorption of $\mathrm{Cr}(\mathrm{VI})$ and $\mathrm{Cr}(\mathrm{III})$, rapid reduction of $\mathrm{Cr}(\mathrm{VI})$ to $\mathrm{Cr}(\mathrm{III})$ in all body fluids and tissues, modest incorporation of chromium into bone, and concentration-dependent urinary clearance consistent with parallel renal processes that conserve chromium efficiently at ambient exposure levels, while the model of Kirman et al. (Kirman et al., 2013) highlighted the notable physiological differences between rodents and humans potentially important for $\mathrm{Cr}(\mathrm{VI})$ reduction.

Polyaromatic hydrocarbons (PAHs) comprise a very large chemical family of more than 46 chemicals that are often found in complex mixture form. For substances of this complex mixture, only two PBTK models were identified; both were related to the reference compound among the various congeners, namely benzo[a]pyrene. The model proposed by Heredia-Ortiz et al., (2014) explicitly described the kinetics of $\mathrm{B}[\mathrm{a}] \mathrm{P}$ transformation into a major metabolite (3Hydroxybenzo(a)pyrene) identified in urine, incorporating all three major exposure routes. On the other hand, the PBTK model of Campbell et al. (2016) was coupled with a more detailed inhalation exposure model for B[a]P that accounts for particle deposition across the human respiratory tract using the multiple-path particle dosimetry (MPPD) model (Anjivel \& Asgharian, 1995). Dissociation of deposited B[a]P from carrier particles was implemented by introducing a first order rate constant by which $\mathrm{B}[\mathrm{a}] \mathrm{P}$ would be transferred to lung tissue.

Regarding PFCs, two models have been recently developed addressing the issue of nonelimination and limited urinary excretion of PFOS and PFOA addressing extrapolation from monkey to humans (Loccisano et al., 2011) and the in uterus and lactation exposure (Loccisano et al., 2013). The model developed by Fabrega et al. (2014) described more explicitly the kinetics of resorption by renal transporters in the filtrate compartment, where chemicals were resorbed from urine back to plasma through a saturable process, while for validation purposes, human data on PFOA and PFOS in human tissues were used. This strategy for estimating tissue:blood partitioning resulted in more accurate model predictions highlighting the advantages of using (ex-vivo) human data over (in vivo) animal data, especially for persistent compounds. 
With regard to phthalates, a PBTK model has been developed by Moreau et al. (2017) for di-2ethylhexyl phthalate (DEHP) and dibutyl phthalate (DnBP) as case compounds. This modelling effort aimed at providing exposure estimates starting from the NHANES biomonitoring data and compared these estimates with high-throughput HT Stochastic Human Exposure and Dose Simulation model (SHEDS-HT) and the ExpoCast heuristic model and non-HT approaches based on chemical specific exposure estimations in the environment in conjunction with human exposure factors. The second model on phthalates by Sharma et al. (2018b) addressed internal dosimetry of DEHP, while for the parameterisation of the model a bottom-up approach was applied, where all the parameters were derived from a combination of in silico (QSAR), in vitro (metabolism) and literature data. In practice, this is a very comprehensive approach for filling the data gaps that are related to PBTK model parameterization, using an in-vitro-to-in-vivo extrapolation (IVIVE) and a quantitative structure-activity relationship (QSAR) method. Moreover, Monte Carlo simulations were performed to estimate the impact of parametric uncertainty onto the model predictions. As a result, model predictions for the DEHP metabolites in both the blood and the urine were well within the range of experimentally observed data.

For brominated flame retardants, no human PBTK model has been identified, so the PBTK model for developmental exposure to BDE-47 in rats (Emond et al., 2010) has been reviewed, as a basis for a future extrapolation to humans, while for aniline and structurally related compounds a PBTK model developed for rat has been extrapolated to humans (Nguyen et al., 2015).

For the mixture of pesticides two models have been found, addressing chlorpyrifos in rats and its extrapolation to humans (Timchalk et al., 2002) and its specific metabolite 3,5,6-trichloro-2pyridinol (TCPY) in young children (Lu et al., 2010).

Finally, five generic PBTK models have been identified, either as stand-alone internal dosimetry models like the IndusChemFate (Jongeneelen and Berge, 2011) and the stochastic PBTK model that was developed by Beaudouin et al. (2010), or incorporated in integrated externalinternal exposure computational platforms such as INTEGRA (Sarigiannis et al., 2014), MENTOR 3P (Xue et al., 2010) and Merlin-Expo (Ciffroy et al., 2016).

It has to be noted that there is a noticeable paucity for available models for the majority of the substances for all substance groups. Availability or paucity of models is governed by (a) the complexity of the chemico-biological interactions and how well these are understood and described / modelled for a given compound and (b) how difficult it is to obtain reliable data for the model parameterisation. However, in many cases, the effort for developing compound specific PBTK models and obtaining data is driven by societal and regulatory needs. The availability of PBTK models for BPA is a good example in this sense; the increased public and regulatory concern in the last eight years about BPA and its potential toxicity during early life exposure resulted in considerable efforts towards the development of the respective PBTK models. Moreover, there are noticeable differences among the developed models, that partially reflect the scientific questions that evolved during the BPA controversy. This explains the comprehensive approach (multi-route exposure, in uterus exposure and lactation) adopted by the Sarigiannis et al. (2016b) model.

How complex should a PBTK model be? Should the complexity of the model be adapted to the specific research question to be addressed? Regarding model structure, it is generally considered that too complex a structure can be deleterious for the accuracy of the model. 
Effectively each parameter value presents a given level of variability and the use of a high number of parameters (in relation to a complex model structure) increases the uncertainty in the model. However, in order to promote the acceptance of PBTK models in the regulatory arena it is critical to move towards generic PBTK models.

Generic PBTK incorporate differences related to inter-individual variability by changing the respective anatomic and physiologic parameters. Efforts to properly parameterise the interactions of the xenobiotics with the given biological systems is a more biology-based approach than trying to progressively approximate the human physiology by introducing ad-hoc compartments aiming to mathematically converge simulation with toxicokinetic data. Thus, the key issue towards PBTK models development and acceptance is parameterisation. For the models presented herein, various parameterisation strategies have been followed including (a) calculations based on in vivo experimental data, (b) calculation from in vitro experimental data, (c) utilisation of values from existing models, (d) fitting of parameters on the basis of experimental data and (e) use of algorithms, namely QSARS and combinations thereof.

Although there is no golden strategy for the derivation of PBTK model parameterisation, given the shift of interest in PBTK models for human risk assessment, model parameterisation strategies relying on human data (in vivo, ex vivo or in vitro) seem to be continuously gaining ground. Towards this aim, the use of targeted HBM campaigns as the ones foreseen in the HBM4EU project, are expected to greatly facilitate the parameterisation process for data poor chemicals, such as bisphenol S and F. On the other hand, computational methods such as QSARs have already been proven to be effective for the parameterisation of toxicokinetic properties (Peyret and Krishnan, 2011). More advanced algorithms that employ machine learning techniques (Ventura et al., 2013) have been shown to be very efficient covering a large chemical space in terms of predicting both metabolic parameters (Sarigiannis et al., 2017) and tissue:blood partition coefficients (Papadaki et al., 2017; Sarigiannis et al., 2017). In addition, existing parameterisation of specific compounds (e.g. BPA) could provide the mechanistic basis of the chemico-biological interactions estimates, based on similarities of the chemical structure. This could greatly facilitate the development of more reliable computationally predicted PBTK parameters of other compounds of the same chemical family, by including the known parameters in the training dataset of the QSAR algorithm.

Finally, a key issue that has to be addressed by future models are mixture interactions. The importance of these interactions is of particular importance for addressing the cumulative exposure regarding the multitude of xenobiotics to which humans are exposed daily (Sarigiannis and Hansen, 2012); this is even more important under occupational exposure. It has been found that for the almost ubiquitous in the ambient and indoor air BTEX mixture, the internal dose of the toxic metabolites in the target tissues is modified by $50 \%$ for exposure levels close to the occupational regulatory limit (threshold limit value, TLV) (Sarigiannis and Gotti, 2008). Similar effects are expected in hot spots where people are exposed to compounds metabolised by the same P450 isophorms, such as PAHs. Moreover, more complex interactions among completely different chemical families have been reported, such as pesticides (atrazine) and heavy metals (arsenic).

With regard to future needs of PBTK modelling, it has to be noted that the advent of the exposome and multi-omics analytical and bioinformatics platforms (Sarigiannis, 2017), as well as the shift of toxicological research to non-animal testing as aptly shown by the large efforts in 
high throughput screening (Hartung et al., 2013), have changed the requirements of PBTK modelling; models are now requested to associate environmentally relevant exposures to molecular responses and to describe exposure-response relationships across new testing (in vitro) systems (Judson et al., 2010; Judson et al., 2011), rather than to calculate tissue doses of in vivo testing. This further supports the need for the development of generic human PBTK models and the need for coordinated strategies for the acquisition of data that will further facilitate successful model parameterisation.

\section{Conclusions}

Physiology based toxicokinetic models provide unique opportunities in modern risk assessment, providing the missing link of external exposure to internal dosimetry, assimilation of biomonitoring data, in vitro testing and molecular epidemiology.

For the first set of prioritised compounds dealt with in the HBM4EU project, the review of the available PBTK models highlights significant conceptual differences between older models compared to the ones developed in the recent years. In the past PBTK models focused on capturing specific chemico-biological interactions that dominate the process of metabolism and elimination; the most recent models, instead, aim at addressing internal dosimetry and the prediction of measured biomarkers under more complex exposure scenarios. However, due to the lack of coordinated strategies for deriving useful biomonitoring data for toxicokinetic properties, significant problems in model parameterisation still remain; these are further increased by the lack of human toxicokinetic data from human volunteers due to ethics issues, as well as to the extent they are really representative of the interindividual variability.

All of the above results in a relative paucity in reliable models for compounds of key regulatory and societal interest, such as bisphenol-F and bisphenol-S, much heralded substitutes for the regulatorily constrained bisphenol-A. QSARs for toxicokinetic properties is an interesting approach to PBTK model parameterisation for data poor chemicals. Overall QSARs should be expanded to model additional chemico-biological interactions, such as intestinal absorption and renal clearance; this could support the development of more complex generic PBTK models, where application to newly derived chemicals should be a problem of parameterisation rather than one of conceptual model development.

Finally, another gap identified is the development of a consistent and computationally efficient framework for mixture interaction among compounds that could eventually interact in terms of metabolism. This is something that could also be addressed by generic PBTK models; however this requires the use of saturable kinetics with regard to the metabolism description.

Concluding all the above and having in mind that that PBTK models aim to serve more precise exposure and risk assessment, it is highly recommended that:

- in terms of conceptual models, efforts are shifted toward the development of generic multi-compartmental and multi-route models,

- development of well-designed and targeted biomonitoring strategies that would efficiently support the accurate parameterisation (in tandem with QSAR-based model estimation, in-vivo and in-vitro data) for newly developed and data poor compounds. 


\section{Acknowledgments}

The authors gratefully acknowledge the financial support of the European Unions' Horizon 2020 research and innovation Programme under grant agreement No 733032 HBM4EU (www.HBM4EU.eu). In addition, they are grateful to Prof. Wilhelm Huisinga of Potsdam University for his useful comments on an early version of the manuscript.

\section{References}

Abraham, K., Mielke, H., Huisinga, W., Gundert-Remy, U., 2005. Elevated internal exposure of children in simulated acute inhalation of volatile organic compounds: effects of concentration and duration. Arch Toxicol 79, 63-73.

Agoram, B., Woltosz, W.S., Bolger, M.B., 2001. Predicting the impact of physiological and biochemical processes on oral drug bioavailability. Adv Drug Deliv Rev 50 Suppl 1, S41-67.

Andra, S.S., Charisiadis, P., Karakitsios, S., Sarigiannis, D.A., Makris, K.C., 2015. Passive exposures of children to volatile trihalomethanes during domestic cleaning activities of their parents. Environmental research 136, 187-195.

Anjilvel, S., Asgharian, B., 1995. A multiple-path model of particle deposition in the rat lung. Fundamental and Applied Toxicology. 28, 41-50.

ATSDR, 1995. Public Health Statement for Polycyclic Aromatic Hydrocarbons (PAHs).

ATSDR, 2002. Aniline. Division of Toxicology ToxFAQs.

ATSDR, 2011. Chromium Toxicity: What Are the Physiologic Effects of Chromium Exposure? Case Studies in Environmental Medicine (CSEM).

ATSDR, 2012. Public Health Statement: Chromium. Division of Toxicology and Human Health Sciences

ATSDR, 2017, Health Effects of PFAS, https://www.atsdr.cdc.gov/pfc/health_effects_pfcs.html,

Beaudouin, R., Micallef, S., Brochot, C., 2010. A stochastic whole-body physiologically based pharmacokinetic model to assess the impact of inter-individual variability on tissue dosimetry over the human lifespan. Regulatory Toxicology and Pharmacology 57, 103-116.

Bechaux, C., Bodin, L., Clemencon, S., Crepet, A., 2014. PBPK and population modelling to interpret urine cadmium concentrations of the French population. Toxicol Appl Pharmacol 279, 364-372.

Bois, F.Y., Jamei, M., Clewell, H.J., 2010. PBPK modelling of inter-individual variability in the pharmacokinetics of environmental chemicals. Toxicology 278, 256-267.

Brochot, C., Quindroit, P., 2018. Modelling the Fate of Chemicals in Humans Using a Lifetime Physiologically Based Pharmacokinetic (PBPK) Model in MERLIN-Expo, in: P. Ciffroy, A. 
Tediosi, E. Capri (Eds.), Modelling the Fate of Chemicals in the Environment and the Human Body. Springer International Publishing, Cham, pp. 215-257.

Campbell, J., Franzen, A., Van Landingham, C., Lumpkin, M., Crowell, S., Meredith, C., Loccisano, A., Gentry, R., Clewell, H., 2016. Predicting lung dosimetry of inhaled particleborne benzo[a]pyrene using physiologically based pharmacokinetic modeling. Inhal Toxicol 28, 520535.

Choi, K., Joo, H., Campbell, J.L., Jr., Clewell, R.A., Andersen, M.E., Clewell, H.J., 3rd, 2012. In vitro metabolism of di(2-ethylhexyl) phthalate (DEHP) by various tissues and cytochrome P450s of human and rat. Toxicology in vitro : an international journal published in association with BIBRA 26, 315-322.

Ciffroy, P., Alfonso, B., Altenpohl, A., Banjac, Z., Bierkens, J., Brochot, C., Critto, A., De Wilde, T., Fait, G., Fierens, T., Garratt, J., Giubilato, E., Grange, E., Johansson, E., Radomyski, A., Reschwann, K., Suciu, N., Tanaka, T., Tediosi, A., Van Holderbeke, M., Verdonck, F., 2016. Modelling the exposure to chemicals for risk assessment: a comprehensive library of multimedia and PBPK models for integration, prediction, uncertainty and sensitivity analysis - the MERLINExpo tool. Science of The Total Environment 568, 770-784.

Clewell, R.A., Clewell, H.J., 3rd, 2008. Development and specification of physiologically based pharmacokinetic models for use in risk assessment. Regulatory toxicology and pharmacology : RTP 50, 129-143.

Clewell, R.A., Kremer, J.J., Williams, C.C., Campbell, J.L., Andersen, M.E., Borghoff, S.J., 2008. Tissue exposures to free and glucuronidated monobutylyphthalate in the pregnant and fetal rat following exposure to di-n-butylphthalate: Evaluation with a PBPK model. Toxicological Sciences 103, 241-259.

Court, M.H., Zhang, X., Ding, X., Yee, K.K., Hesse, L.M., Finel, M., 2012. Quantitative distribution of mRNAs encoding the 19 human UDP-glucuronosyltransferase enzymes in 26 adult and 3 fetal tissues. Xenobiotica 42, 266-277.

Crowell, S.R., Amin, S.G., Anderson, K.A., Krishnegowda, G., Sharma, A.K., Soelberg, J.J., Williams, D.E., Corley, R.A., 2011. Preliminary physiologically based pharmacokinetic models for benzo[a]pyrene and dibenzo[def,p]chrysene in rodents. Toxicol Appl Pharmacol 257, 365376.

Csanady, G.A., Oberste-Frielinghaus, H.R., Semder, B., Baur, C., Schneider, K.T., Filser, J.G., 2002. Distribution and unspecific protein binding of the xenoestrogens bisphenol $A$ and daidzein. Arch Toxicol 76, 299-305.

Domingo, J.L., 2012. Health risks of dietary exposure to perfluorinated compounds. Environ Int 40, 187-195.

Edginton, A.N., Ritter, L., 2009. Predicting plasma concentrations of bisphenol A in children younger than 2 years of age after typical feeding schedules, using a physiologically based toxicokinetic model. Environmental Health Perspectives 117, 645-652.

ECHA, Inclusion of substances of very high concern in the Candidate List for eventual inclusion in Annex XIV. 03.01.2018, TEM-0013.08., Helsinki, 2018. 
Edginton, A.N., Schmitt, W., Voith, B., Willmann, S., 2006. A mechanistic approach for the scaling of clearance in children. Clin Pharmacokinet 45, 683-704.

EFSA, 2015. Scientific Opinion on the risks to public health related to the presence of bisphenol A (BPA) in foodstuffs: Executive summary.

Eissing, T., Kuepfer, L., Becker, C., Block, M., Coboeken, K., Gaub, T., Goerlitz, L., Jaeger, J., Loosen, R., Ludewig, B., Meyer, M., Niederalt, C., Sevestre, M., Siegmund, H.U., Solodenko, J., Thelen, K., Telle, U., Weiss, W., Wendl, T., Willmann, S., Lippert, J., 2011. A computational systems biology software platform for multiscale modeling and simulation: Integrating wholebody physiology, disease biology, and molecular reaction networks. Frontiers in Physiology FEB.

Emond, C., Raymer, J.H., Studabaker, W.B., Garner, C.E., Birnbaum, L.S., 2010. A physiologically based pharmacokinetic model for developmental exposure to BDE-47 in rats. Toxicology and Applied Pharmacology 242, 290-298.

Fabrega, F., Kumar, V., Schuhmacher, M., Domingo, J.L., Nadal, M., 2014. PBPK modeling for PFOS and PFOA: validation with human experimental data. Toxicol Lett 230, 244-251.

FDA, 2014. Bisphenol A (BPA): Use in Food Contact Application.

Fromme, H., Becher, G., Hilger, B., Völkel, W., 2016. Brominated flame retardants - Exposure and risk assessment for the general population. International Journal of Hygiene and Environmental Health. 219, 1-23.

Fisher, J.W., Twaddle, N.C., Vanlandingham, M., Doerge, D.R., 2011. Pharmacokinetic modeling: prediction and evaluation of route dependent dosimetry of bisphenol $A$ in monkeys with extrapolation to humans. Toxicol Appl Pharmacol 257, 122-136.

Ganzleben, C., Antignac, J.P., Barouki, R., Castano, A., Fiddicke, U., Klanova, J., Lebret, E., Olea, N., Sarigiannis, D., Schoeters, G.R., Sepai, O., Tolonen, H., Kolossa-Gehring, M., 2017. Human biomonitoring as a tool to support chemicals regulation in the European Union. Int J Hyg Environ Health 220, 94-97.

Gauderat, G., Picard-Hagen, N., Toutain, P.-L., Servien, R., Viguié, C., Puel, S., Lacroix, M. Z., Corbel, T., Bousquet-Melou, A., Gayrard, V., 2017. Prediction of human prenatal exposure to bisphenol A and bisphenol A glucuronide from an ovine semi-physiological toxicokinetic model. Scientific reports. 7, 15330-15330.

Gentry, P. R., H. J. Clewell, R. Clewell, J. Campbell, C. Van Landingham \& A. M. Shipp (2011) Challenges in the application of quantitative approaches in risk assessment: a case study with di-(2-ethylhexyl)phthalate. Critical Reviews in Toxicology, 41, 1-72.

Georgopoulos, P.G., Lioy, P.J., 2006. From a theoretical framework of human exposure and dose assessment to computational system implementation: the Modeling ENvironment for TOtal Risk Studies (MENTOR). Journal of toxicology and environmental health. Part B, Critical reviews 9 , 457-483.

Georgopoulos, P.G., Sasso, A.F., Isukapalli, S.S., Lioy, P.J., Vallero, D.A., Okino, M., Reiter, L., 2008a. Reconstructing population exposures to environmental chemicals from biomarkers: challenges and opportunities. Journal of Exposure Science and Environmental Epidemiology 19, 149-171. 
Georgopoulos, P.G., Wang, S.W., Yang, Y.C., Xue, J., Zartarian, V.G., McCurdy, T., Ozkaynak, H., 2008b. Biologically based modeling of multimedia, multipathway, multiroute population exposures to arsenic. Journal of Exposure Science and Environmental Epidemiology 18, 462476.

Gilden, R.C., Huffling, K., Sattler, B., 2010. Pesticides and health risks. Journal of obstetric, gynecologic, and neonatal nursing : JOGNN 39, 103-110.

Harrison, J.H., Jr., Jollow, D.J., 1987. Contribution of aniline metabolites to aniline-induced methemoglobinemia. Mol Pharmacol 32, 423-431.

Hartung, T., Luechtefeld, T., Maertens, A., Kleensang, A., 2013. Integrated testing strategies for safety assessments. Altex 30, 3-18.

Heredia-Ortiz, R., Bouchard, M., 2013. Understanding the linked kinetics of benzo(a)pyrene and 3-hydroxybenzo(a)pyrene biomarker of exposure using physiologically-based pharmacokinetic modelling in rats. Journal of Pharmacokinetics and Pharmacodynamics 40, 669-682.

Heredia-Ortiz, R., Maitre, A., Barbeau, D., Lafontaine, M., Bouchard, M., 2014. Use of Physiologically-Based Pharmacokinetic Modeling to Simulate the Profiles of 3-Hydroxybenzo(a) pyrene in Workers Exposed to Polycyclic Aromatic Hydrocarbons. Plos One 9, e102570.

Heudorf, U., Mersch-Sundermann, V., Angerer, J., 2007. Phthalates: Toxicology and exposure. International Journal of Hygiene and Environmental Health 210, 623-634.

Jarup, L., Akesson, A., 2009. Current status of cadmium as an environmental health problem. Toxicol Appl Pharmacol 238, 201-208.

Johnson, J.D., Gibson, S.J., Ober, R.E., 1984. Cholestyramine-enhanced fecal elimination of carbon-14 in rats after administration of ammonium [14C]perfluorooctanoate or potassium [14C]perfluorooctanesulfonate. Fundam Appl Toxicol 4, 972-976.

Jongeneelen, F.J., Berge, W.F.T., 2011. A generic, cross-chemical predictive PBTK model with multiple entry routes running as application in MS Excel; design of the model and comparison of predictions with experimental results. Annals of Occupational Hygiene 55, 841-864.

Jongeneelen, F.J., Ten Berge, W.F., 2011. A Generic, Cross-Chemical Predictive PBTK Model with Multiple Entry Routes Running as Application in MS Excel; Design of the Model and Comparison of Predictions with Experimental Results. Annals of Occupational Hygiene 55, 841864.

Judson, R.S., Houck, K.A., Kavlock, R.J., Knudsen, T.B., Martin, M.T., Mortensen, H.M., Reif, D.M., Rotroff, D.M., Shah, I., Richard, A.M., Dix, D.J., 2010. In vitro screening of environmental chemicals for targeted testing prioritization: The toxcast project. Environmental Health Perspectives 118, 485-492.

Judson, R.S., Kavlock, R.J., Setzer, R.W., Cohen Hubal, E.A., Martin, M.T., Knudsen, T.B., Houck, K.A., Thomas, R.S., Wetmore, B.A., Dix, D.J., 2011. Estimating toxicity-related biological pathway altering doses for high-throughput chemical risk assessment. Chemical Research in Toxicology 24, 451-462.

Kamrin, M.A., 2009. Phthalate risks, phthalate regulation, and public health: a review. Journal of toxicology and environmental health. Part B, Critical reviews 12, 157-174. 
Kearney, T.E., Manoguerra, A.S., Dunford, J.V., 1984. Chemically induced methemoglobinemia from aniline poisoning. Western Journal of Medicine 140, 282-286.

Keith, L.H., 1997. Environmental Endocrine Disruptors: A Handbook of Property Data. Wiley.

Kirman, C.R., Aylward, L.L., Suh, M., Harris, M.A., Thompson, C.M., Haws, L.C., Proctor, D.M., Lin, S.S., Parker, W., Hays, S.M., 2013. Physiologically based pharmacokinetic model for humans orally exposed to chromium. Chemico-Biological Interactions 204, 13-27.

Kirman, C.R., Hays, S.M., Aylward, L.L., Suh, M., Harris, M.A., Thompson, C.M., Haws, L.C., Proctor, D.M., 2012. Physiologically based pharmacokinetic model for rats and mice orally exposed to chromium. Chem Biol Interact 200, 45-64.

Kjellström, T., Nordberg, G.F., 1978. A kinetic model of cadmium metabolism in the human being. Environmental Research 16, 248-269.

Klaassen, C.D., 2007. Casarett \& Doull's Toxicology: The Basic Science of Poisons, Seventh Edition. McGraw-Hill Education.

Krauss, M., Schaller, S., Borchers, S., Findeisen, R., Lippert, J., Kuepfer, L., 2012. Integrating Cellular Metabolism into a Multiscale Whole-Body Model. PLoS Computational Biology 8.

Kurebayashi, H., Harada, R., Stewart, R.K., Numata, H., Ohno, Y., 2002. Disposition of a low dose of bisphenol a in male and female cynomolgus monkeys. Toxicol Sci 68, 32-42.

Kurebayashi, H., Okudaira, K., Ohno, Y., 2010. Species difference of metabolic clearance of bisphenol A using cryopreserved hepatocytes from rats, monkeys and humans. Toxicology Letters 198, 210-215.

Langard, S., 1990. One hundred years of chromium and cancer: a review of epidemiological evidence and selected case reports. Am J Ind Med 17, 189-215.

Lee, C.H., Kim, S.H., Kwon, D.H., Jang, K.H., Chung, Y.H., Moon, J.D., 2013. Two Cases of Methemoglobinemia Induced by the Exposure to Nitrobenzene and Aniline. Annals of Occupational and Environmental Medicine 25, 31-31.

Lee, S.K., Ou, Y.C., Andersen, M.E., Yang, R.S.H., 2007. A physiologically based pharmacokinetic model for lactational transfer of PCB 153 with or without PCB 126 in mice. Archives of Toxicology 81, 101-111.

Leeder, J.S., 2009. Developmental pharmacogenetics: A general paradigm for application to neonatal pharmacology and toxicology. Clinical Pharmacology and Therapeutics 86, 678-682.

Loccisano, A.E., Campbell, J.L., Andersen, M.E., Clewell, H.J., 2011. Evaluation and prediction of pharmacokinetics of PFOA and PFOS in the monkey and human using a PBPK model. Regulatory Toxicology and Pharmacology 59, 157-175.

Loccisano, A.E., Campbell, J.L., Jr., Butenhoff, J.L., Andersen, M.E., Clewell, H.J., 3rd, 2012. Evaluation of placental and lactational pharmacokinetics of PFOA and PFOS in the pregnant, lactating, fetal and neonatal rat using a physiologically based pharmacokinetic model. Reprod Toxicol 33, 468-490. 
Loccisano, A.E., Longnecker, M.P., Campbell, J.L., Andersen, M.E., Clewell, H.J., 2013.

Development of pbpk models for pfoa and pfos for human pregnancy and lactation life stages. Journal of Toxicology and Environmental Health - Part A: Current Issues 76, 25-57.

Loizou, G., Spendiff, M., Barton, H.A., Bessems, J., Bois, F.Y., d'Yvoire, M.B., Buist, H., Clewell, H.J., Meek, B., Gundert-Remy, U., Goerlitz, G., Schmitt, W., 2008. Development of good modelling practice for physiologically based pharmacokinetic models for use in risk assessment: The first steps. Regulatory Toxicology and Pharmacology 50, 400-411.

Lu, C., Holbrook, C.M., Andres, L.M., 2010. The implications of using a physiologically based pharmacokinetic (PBPK) model for pesticide risk assessment. Environ Health Perspect 118, 125-130.

Mandiwana, K.L., Panichev, N., Panicheva, S., 2011. Determination of chromium(VI) in black, green and herbal teas. Food Chemistry 129, 1839-1843.

Meeker, J.D., Sathyanarayana, S., Swan, S.H., 2009. Phthalates and other additives in plastics: human exposure and associated health outcomes. Philosophical transactions of the Royal Society of London. Series B, Biological sciences 364, 2097-2113.

Mielke, H., Gundert-Remy, U., 2009. Bisphenol A levels in blood depend on age and exposure. Toxicology Letters 190, 32-40.

Mills, C.T., Morrison, J.M., Goldhaber, M.B., Ellefsen, K.J., 2011. Chromium(VI) generation in vadose zone soils and alluvial sediments of the southwestern Sacramento Valley, California: a potential source of geogenic $\mathrm{Cr}(\mathrm{VI})$ to groundwater. Applied Geochemistry 26, 1488-1501.

Minoia, C., Cavalleri, A., 1988. Chromium in urine, serum and red blood cells in the biological monitoring of workers exposed to different chromium valency states. Sci Total Environ 71, 323327.

Mitragotri, S., Anissimov, Y.G., Bunge, A.L., Frasch, H.F., Guy, R.H., Hadgraft, J., Kasting, G.B., Lane, M.E., Roberts, M.S., 2011. Mathematical models of skin permeability: An overview. International Journal of Pharmaceutics 418, 115-129.

Moreau, M., Leonard, J., Phillips, K.A., Campbell, J., Pendse, S.N., Nicolas, C., Phillips, M., Yoon, M., Tan, Y.M., Smith, S., Pudukodu, H., Isaacs, K., Clewell, H., 2017. Using exposure prediction tools to link exposure and dosimetry for risk-based decisions: A case study with phthalates. Chemosphere 184, 1194-1201.

National Research Council, 2008. Phthalates and Cumulative Risk Assessment: The Tasks Ahead. The National Academies Press, Washington, DC.

Nguyen, H.Q., Stamatis, S.D., Kirsch, L.E., 2015. A Novel Method for Assessing Drug Degradation Product Safety Using Physiologically-Based Pharmacokinetic Models and Stochastic Risk Assessment. Journal of Pharmaceutical Sciences 104, 3101-3119.

Nicolopoulou-Stamati, P., Maipas, S., Kotampasi, C., Stamatis, P., Hens, L., 2016. Chemical Pesticides and Human Health: The Urgent Need for a New Concept in Agriculture. Frontiers in Public Health 4, 148.

Nordberg, G.F., Fowler, B.A., Nordberg, M., 2014. Handbook on the Toxicology of Metals. Elsevier Science. 
O'Flaherty, E.J., 1993. Physiologically based models for bone-seeking elements. IV. Kinetics of lead disposition in humans. Toxicol Appl Pharmacol 118, 16-29.

O'Flaherty, E.J., 1996. A physiologically based model of chromium kinetics in the rat. Toxicol Appl Pharmacol 138, 54-64.

O'Flaherty, E.J., Kerger, B.D., Hays, S.M., Paustenbach, D.J., 2001. A physiologically based model for the ingestion of chromium(III) and chromium(VI) by humans. Toxicological sciences : an official journal of the Society of Toxicology 60, 196-213.

O’Flaherty, E.J., 1995. Chromium Toxicokinetics, in: R.A. Goyer, M.G. Cherian (Eds.), Toxicology of Metals: Biochemical Aspects. Springer Berlin Heidelberg, Berlin, Heidelberg, pp. 215-228.

Oze, C., Bird, D.K., Fendorf, S., 2007. Genesis of hexavalent chromium from natural sources in soil and groundwater. Proc Natl Acad Sci U S A 104, 6544-6549.

Papadaki, K.C., Karakitsios, S.P., Sarigiannis, D.A., 2017. Modeling of adipose/blood partition coefficient for environmental chemicals. Food and Chemical Toxicology 110, 274-285.

Peyret, T., Krishnan, K., 2011. QSARs for PBPK modelling of environmental contaminants. SAR and QSAR in Environmental Research 22, 129-169.

Price, K., Krishnan, K., 2011. An integrated QSAR-PBPK modelling approach for predicting the inhalation toxicokinetics of mixtures of volatile organic chemicals in the rat. SAR and QSAR in Environmental Research 22, 107-128.

Ramsey, J.C., Andersen, M.E., 1984. A physiologically based description of the inhalation pharmacokinetics of styrene in rats and humans. Toxicology and Applied Pharmacology 73, 159-175.

Ruiz, P., Fowler, B.A., Osterloh, J.D., Fisher, J., Mumtaz, M., 2010. Physiologically based pharmacokinetic (PBPK) tool kit for environmental pollutants--metals. SAR QSAR Environ Res 21, 603-618.

Sanborn, M., Kerr, K.J., Sanin, L.H., Cole, D.C., Bassil, K.L., Vakil, C., 2007. Non-cancer health effects of pesticides: systematic review and implications for family doctors. Canadian family physician Medecin de famille canadien 53, 1712-1720.

Sarigiannis, D., Gotti, A., Karakitsios, S., 2011. A Computational Framework for Aggregate and Cumulative Exposure Assessment. Epidemiology 22, S96-S97.

Sarigiannis, D., Karakitsios, S., 2011. Perinatal Exposure to Bisphenol A: The Route of Administration Makes the Dose. Epidemiology 22, S172.

Sarigiannis, D., Karakitsios, S., Gotti, A., Loizou, G., Cherrie, J., Smolders, R., De Brouwere, K., Galea, K., Jones, K., Handakas, E., Papadaki, K., Sleeuwenhoek, A., 2014. Integra: From global scale contamination to tissue dose, Proceedings - 7th International Congress on Environmental Modelling and Software: Bold Visions for Environmental Modeling, iEMSs 2014, pp. 1001-1008.

Sarigiannis, D.A., 2017. Assessing the impact of hazardous waste on children's health: The exposome paradigm. Environ Res 158, 531-541. 
Sarigiannis, D.A., Gotti, A., 2008. Biology-based dose-response models for health risk assessment of chemical mixtures. Fresenius Environmental Bulletin 17, 1439-1451.

Sarigiannis, D.A., Hansen, U., 2012. Considering the cumulative risk of mixtures of chemicals A challenge for policy makers. Environmental Health: A Global Access Science Source 11.

Sarigiannis, D.A., Karakitsios, S.P., 2012. A dynamic physiology based pharmacokinetic model for assessing lifelong internal dose, AIChE 2012, Pittsburgh, PA.

Sarigiannis, D.A., Karakitsios, S.P., Handakas, E., Simou, K., Solomou, E., Gotti, A., 2016. Integrated exposure and risk characterization of bisphenol-A in Europe. Food and Chemical Toxicology 98, 134-147.

Sarigiannis, D.A., Papadaki, K., Kontoroupis, P., Karakitsios, S.P., 2017. Development of QSARs for parameterizing Physiology Based ToxicoKinetic models. Food and Chemical Toxicology 106, 114-124.

Sarigiannis, D.A., Karakitsios, S.P., Zikopoulos, D., Nikolaki, S., Kermenidou, M., 2015. Lung cancer risk from PAHs emitted from biomass combustion. Environmental Research. 137, 147156.

Sasso, A.F., Isukapalli, S.S., Georgopoulos, P.G., 2010. A generalized physiologically-based toxicokinetic modeling system for chemical mixtures containing metals. Theoretical Biology and Medical Modelling 7.

Satarug, S., Garrett, S.H., Sens, M.A., Sens, D.A., 2010. Cadmium, environmental exposure, and health outcomes. Environ Health Perspect 118, 182-190.

Sharma, R.P., Schuhmacher, M., Kumar, V., 2018a. The development of a pregnancy PBPK Model for Bisphenol A and its evaluation with the available biomonitoring data. Science of Total Environment in press.

Sharma, R.P., Schuhmacher, M., Kumar, V., 2018b. Physiology based Pharmacokinetic (PBPK) modeling for DEHP metabolites integrating its in vitro metabolism: a bottom up modeling approach. Toxicology Letters Submitted.

Shin, B.S., Kim, C.H., Jun, Y.S., Kim, D.H., Lee, B.M., Yoon, C.H., Park, E.H., Lee, B.M., Yoon, C.H., Park, E.H., Lee, K.C., Han, S.Y., Park, K.L., Kim, H.S., Yoo, S.D., 2004. Physiologically based pharmacokinetics of bisphenol A. Journal of Toxicology and Environmental Health - Part A 67, 1971-1985.

Stern, A.H., 2010. A quantitative assessment of the carcinogenicity of hexavalent chromium by the oral route and its relevance to human exposure. Environ Res 110, 798-807.

Swan, S.H., 2008. Environmental phthalate exposure in relation to reproductive outcomes and other health endpoints in humans. Environmental research 108, 177-184.

Tan, Y.M., Liao, K., Conolly, R., Blount, B., Mason, A., Clewell, H., 2006. Use of a physiologically based pharmacokinetic model to identify exposures consistent with human biomonitoring data for chloroform. Journal of Toxicology and Environmental Health - Part A: Current Issues 69, 1727-1756.

Teeguarden, J.G., Waechter, J.M., Clewell, H.J., Covington, T.R., Barton, H.A., 2005.

Evaluation of oral and intravenous route pharmacokinetics, plasma protein binding, and uterine 
tissue dose metrics of bisphenol A: A physiologically based pharmacokinetic approach. Toxicological Sciences 85, 823-838.

ten Berge, W., 2009. A simple dermal absorption model: derivation and application. Chemosphere 75, 1440-1445.

Thayer, K.A., Doerge, D.R., Hunt, D., Schurman, S.H., Twaddle, N.C., Churchwell, M.I., Garantziotis, S., Kissling, G.E., Easterling, M.R., Bucher, J.R., Birnbaum, L.S., 2015. Pharmacokinetics of Bisphenol A in Humans Following a Single Oral Administration. Environment international 83, 107-115.

Timchalk, C., Nolan, R.J., Mendrala, A.L., Dittenber, D.A., Brzak, K.A., Mattsson, J.L., 2002. A Physiologically based pharmacokinetic and pharmacodynamic (PBPK/PD) model for the organophosphate insecticide chlorpyrifos in rats and humans. Toxicological sciences : an official journal of the Society of Toxicology 66, 34-53.

Touitou, E., 2002. Drug delivery across the skin. Expert Opinion on Biological Therapy 2, 723733.

USEPA, 2013. Provisional Guidance for Quantitative Risk Assessment of Polycyclic Aromatic Hydrocarbons.

Valcke, M., Krishnan, K., 2011. Evaluation of the impact of the exposure route on the human kinetic adjustment factor. Regulatory Toxicology and Pharmacology 59, 258-269.

Vanden Heuvel, J.P., Kuslikis, B.I., Van Rafelghem, M.J., Peterson, R.E., 1991. Tissue distribution, metabolism, and elimination of perfluorooctanoic acid in male and female rats. Journal of biochemical toxicology 6, 83-92.

Ventura, C., Latino, D.A.R.S., Martins, F., 2013. Comparison of Multiple Linear Regressions and Neural Networks based QSAR models for the design of new antitubercular compounds. European journal of medicinal chemistry 70, 831-845.

Verner, M.A., Charbonneau, M., Lopez-Carrillo, L., Haddad, S., 2008. Physiologically based pharmacokinetic modeling of persistent organic pollutants for lifetime exposure assessment: A new tool in breast cancer epidemiologic studies. Environmental Health Perspectives 116, 886892.

Wagner, J.G., 1973. Properties of Michaelis-Menten equation and its integrated form which are useful in pharmacokinetics. Journal of Pharmacokinetics and Biopharmaceutics 1, 103-121.

WHO, 2010. Exposure to highly hazardous pesticides: A major public health concern, Geneva, Switzerland.

Willhite, C.C., Ball, G.L., McLellan, C.J., 2008. Derivation of a bisphenol A oral reference dose $(\mathrm{RfD})$ and drinking-water equivalent concentration. Journal of toxicology and environmental health. Part B, Critical reviews 11, 69-146.

Willmann, S., Lippert, J., Sevestre, M., Solodenko, J., Fois, F., Schmitt, W., 2003. PK-Sim®: A physiologically based pharmacokinetic 'whole-body' model. Drug Discovery Today: BIOSILICO 1, 121-124. 
Willmann, S., Schmitt, W., Keldenich, J., Lippert, J., Dressman, J.B., 2004. A physiological model for the estimation of the fraction dose absorbed in humans. Journal of Medicinal Chemistry 47, 4022-4031.

World Health Organization, 2010. WHO Guidelines for Indoor Air Quality: Selected Pollutants. WHO.

Xue, J., Zartarian, V., Wang, S.W., Liu, S.V., Georgopoulos, P., 2010. Probabilistic modeling of dietary arsenic exposure and dose and evaluation with 2003-2004 NHANES data.

Environmental Health Perspectives 118, 345-350.

Yang, X., Doerge, D.R., Teeguarden, J.G., Fisher, J.W., 2015. Development of a physiologically based pharmacokinetic model for assessment of human exposure to bisphenol A. Toxicol Appl Pharmacol 289, 442-456.

Yang, Y., Xu, X., Georgopoulos, P.G., 2010. A Bayesian population PBPK model for multiroute chloroform exposure. Journal of Exposure Science and Environmental Epidemiology 20, 326341.

Yu, L.X., Amidon, G.L., 1999. A compartmental absorption and transit model for estimating oral drug absorption. International journal of pharmaceutics 186, 119-125. 